\title{
The stochastic multiplicative cascade structure of deterministic numerical models of the atmosphere
}

\author{
J. Stolle ${ }^{1}$, S. Lovejoy ${ }^{1}$, and D. Schertzer ${ }^{2,3}$ \\ ${ }^{1}$ Physics, McGill University, 3600 University St., Montreal, Que. H3A 2T8, Canada \\ ${ }^{2}$ CEREVE, Université Paris Est, Marne-la-Vallée, France \\ ${ }^{3}$ Météo France, 1 Quai Branly, Paris 75005, France
}

Received: 16 February 2009 - Revised: 1 July 2009 - Accepted: 22 August 2009 - Published: 26 October 2009

\begin{abstract}
By direct statistical analysis we show that over almost all their range of scales and to within typically better than $\pm 1 \%$, atmospheric fields obtained from analyses and numerical integrations of atmospheric models have the multifractal structure predicted by multiplicative cascade models. We quantify this for the horizontal wind, temperature, and humidity fields at 5 different pressure levels for the ERA40 reanalysis, the Canadian Meteorological Centre Global Environmental Multiscale (CMC, GEM) model, as well as the National Oceanographic and Atmospheric Administration Global Forecasting System (NOAA, GFS). We investigate the additional prediction that the cascade belongs to a universal multifractal basin of attraction. By demonstrating a "Levy collapse" of the statistical moments to within \pm 2 to $\pm 5 \%$ over most of the range of scales, we conclude that there is good evidence for this. Finally, we discuss how this stochastic multiplicative cascade structure can be exploited in improving ensemble forecasts.
\end{abstract}

\section{Introduction}

\subsection{Numerical weather prediction and cascades}

Richardson's seminal book "Weather prediction by numerical process" (1922) is venerated as the pioneering work in numerical atmospheric modelling. With a lapse of about 30 years, Richardson's idea was taken up by generations of atmospheric scientists; the result is modern ensemble forecasting systems. However in the same book, and in a subsequent papers (especially Richardson, 1926), the seed of another idea was sown: that atmospheric dynamics might repeat scale by scale in a cascade-like manner. In other words

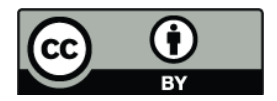

Correspondence to: J. Stolle

(stollej@physics.mcgill.ca)
Richardson suspected that - if viewed correctly - the seeming complexity of the brute force numerics might hide scale by scale simplicity.

In the case of cascades, he was about 40 years ahead of his time: it was not until the 1960's, that explicit multiplicative cascade models were first developed (Novikov and Stewart, 1964; Yaglom, 1966; Mandelbrot, 1974). These models are based on the scale symmetry (broken only by viscosity at small scales and the forcing at large scales), by scale conservation of energy flux and the Fourier localness of the nonlinear interactions (so that structures of a given scale mostly interact with other structures of similar scale). These multiplicative cascades can be understood as attempts to deduce the implications of some (but not all) of the symmetries of the governing equations. Since the mid 1980's it was realized that multiplicative cascade processes are very general; they are the generic multifractal process. Today, their nontrivial statistical properties are relatively well understood and they have been applied throughout physics and the geosciences.

\subsection{Cascades in geophysical turbulence}

While cascades have regularly been invoked in laboratory, geophysical, and astrophysical turbulence, the appropriate turbulent cascade flux (e.g. energy, enstrophy etc.) is typically determined a priori by theoretical considerations. Subsequent quantitative cascade tests are usually limited to the comparison of numerically or empirically determined spectral exponents with those expected (on essentially dimensional grounds) for the given (supposedly dominant) turbulent fluxes. The most important classical spectral exponents are $-5 / 3,-3$ for the velocity exponents in regimes dominated by energy and enstrophy fluxes respectively. For example, in analyzing atmospheric models, Steinberg et al. (1971), Boer and Shepherd (1983), and Straus and Ditlevsen (1999) calculated spectra and spectral transfers of energy, enstrophy and pseudo-potential enstrophy. The latter paper is particularly pertinent to our discussion since the large atmospheric

Published by Copernicus Publications on behalf of the European Geosciences Union and the American Geophysical Union. 
"reanalyses" that it uses are earlier and slightly lower resolution versions of some of the data analysed here. The authors came to some strong conclusions: that there was no evidence for $k^{-5 / 3}$ or $k^{-3}$ behaviours nor for any upscale transient energy transfer (the usual signature of 2-D turbulence).

Whereas the great majority of turbulence theories are isotropic - or at least quasi isotropic (they have the same exponents but not necessarily the same prefactors in all directions) - empirical studies of the vertical atmospheric structure, e.g., Van Zandt (1982), Schertzer and Lovejoy (1985), Dewan and Good (1986), Gardner (1994), Dewan (1997), Lilley et al. (2004), Lilley et al. (2008), show on the contrary that the turbulence is anisotropic, with vertical exponents different from those in the horizontal so that the stratification is scaling. They therefore require anisotropic theories such as the quasi-linear gravity wave theories - e.g. the Saturated Cascade Theory (Dewan and Good, 1986; Dewan, 1997) or the Diffusive Filtering Theory (Gardner, 1994) or the strongly nonlinear 23/9D buoyancy flux/energy flux model (Schertzer and Lovejoy, 1985).

Recent analyses of massive quantities of global scale satellite data (visible, infra-red, passive and active microwave wavelengths) have provided new impetus for attempting to test multiplicative cascades on atmospheric models. These studies show empirically that to within $\approx \pm 1 \%$, the energy containing short and long wave atmospheric radiances respect the predictions of multiplicative cascades from planetary scales down to at least several kilometres (Lovejoy et al., 2001, 2009a) so that the relevant sources and sinks of fluxes are likely to be scaling. Similarly, Lovejoy et al. (2009c, d) showed that the vertical structure of horizontal wind, passive scalars, temperature, pressure, humidity, potential temperature, etc. also have cascade structures down to $<\approx 5 \mathrm{~m}$ with outer scales in the range $1-30 \mathrm{~km}$ (depending on the field). The picture that emerges from this wide range and anisotropic cascade structure is at odds with the standard model of atmospheric dynamics which involves a "dimensional transition" between isotropic 3-D and isotropic 2-D turbulence (the "meso-scale gap"). Indeed, a recent survey (and criticism) of empirical studies over the last 30 years (Lilley et al., 2004, 2008; Lovejoy et al., 2008) shows that the classical studies of Gage and Nastrom (1986) (the GASP experiment) and Cho and Lindborg (2001) (MOZAIC) must be reconsidered, especially their conclusions about spectral breaks and the 2-D turbulence nature of the large scales. The key to this re-evaluation is partly the use of much higher quality modern data (especially from lidar and drop sondes), but also the demonstration that in anisotropic (but scaling) turbulence that the nature of aircraft trajectories - whether on isomachs, isobars, or isoheights), as well as the effect of their vertical fluctuations must be more carefully taken into account. For example, vertical fluctuations of the aircraft may themselves lead to spurious scale breaks and spurious exponents (the stratosphere; Lovejoy et al., 2004). Alternatively, the exponents obtained from aircraft flying along isobars - as they invariably do in the troposphere for air traffic control reasons - can yield exponents spuriously close to the vertical (rather than horizontal) values; they yield spectra exponents of $\approx-2.4$ (rather than $\approx-5 / 3$ ) at large scales (Lovejoy et al., 2009c). It is interesting to note that the models discussed here are all hydrostatic so that the "horizontal" levels we analyze are actually isobars rather than isoheights. The fact that the wind spectra along isobars and isoheights are quite different $\left(k^{-2.4}\right.$ rather than $\left.k^{-5 / 3}\right)$ is important and helps explain the model statistics - which for the wind is also close to $k^{-2.4}$ on isobars; this will be discussed elsewhere.

If the velocity field has a scaling cascade structure, then we expect that there is also a temporal cascade structure so that the cascades are actually in space-time. We confirm this in a forthcoming publication, where we discuss the relationship between the spatial and temporal structures (Stolle, 2009; Stolle et al., 2009). Lovejoy and Schertzer (2009) gives a recent review of some of this work showing the ubiquity of space-time cascade structures and arguing that it allows for a new synthesis of nonlinear dynamics with state of the art atmospheric data. To summarize: it now seems that for the models to be realistic, they must have cascade structures, too.

\subsection{Cascades in direct numerical simulations}

Before turning our attention to the analysis of atmospheric models, we should also mention the related field of pure hydrodynamic turbulence which is often considered more fundamental than atmospheric turbulence, but where the same issues have arisen. They too can involve structures spanning huge ranges of scale and despite intense efforts over more than 50 years, analytic approaches have been largely ineffective; given their successes elsewhere in fundamental physics, this is perhaps surprising. The limitations of statistical closure, renormalization, and other kindred analytical techniques have lead to the development of two main alternatives: brute force numerics and phenomenological models, especially cascades.

Although Direct Numerical Simulations (DNS; i.e. without subgrid "parameterizations") of Navier Stokes (NS) equations have been made since Orszag and Patterson (1972), it was not until Vincent and Meneguzzi (1991) that computers were powerful enough to allow for simulations large enough to display hints of the Kolmogorov $k^{-5 / 3}$ spectra, the traditional signature of the inertial range. The DNS inertial range is limited primarily because the dissipation is usually modelled with a Laplacian operator that is typically significant over a range a large factor $(\approx 50)$ in scale. Recent Earth Simulator integrations on a $4096^{3}$ grid are now able to display roughly an intermediate (inertial) range spanning a factor of $\approx 100$ in scale (Yokokawa et al., 2002; Kaneda, 2003), but require massive computational efforts. 


\subsection{Goals and structure}

The aim of this paper is thus to test the predictions of multiplicative cascades on the spatial structure of various numerical models of the atmosphere. This paper is structured as follows. In Sect. 2 we review the basic theory including the predictions of the multiplicative models and different methods of estimating fluxes. In Sect. 3 we describe the data sets and present the basic results and in Sect. 4 we conclude.

\section{Multiplicative cascade models}

\subsection{Basic statistics}

During the 1960's and early 1970's, intermittency was increasingly acknowledged as an important phenomenon, but its effect was usually considered small, associated primarily with small corrections to the spectral exponents. The main statistical models (such as those used in statistical closures) assumed "quasi-Gaussian statistics". In order to obtain a Gaussian model with the classical Kolmogorov law $\Delta v=\varepsilon^{1 / 3} \Delta x^{1 / 3}$ (for velocity fluctuations $\Delta v$ over distances $\Delta x$ ) - the real space equivalent of what is given in Fourier space in Kolmogorov (1941) -, it is sufficient to take the energy flux $\varepsilon$ as a Gaussian white noise process and give $\varepsilon^{1 / 3}$ a (fractional) integration of order $1 / 3$ (i.e., a power law filter of order $-1 / 3$ ): the resulting $v$ is a "fractional Brownian motion".

In order to take into account intermittency, it suffices to replace the Gaussian $\varepsilon$ in the above model by the result of a multiplicative cascade; this is the Fractionally Integrated Flux model (Schertzer and Lovejoy, 1987). In multiplicative cascades, large structures are broken up into smaller daughter structures which multiplicatively modulate the flux; this process is repeated to smaller and smaller scales. Normalized cascade processes generally lead to multifractal fields with statistics:

$$
\left\langle\varphi_{\lambda}^{q}\right\rangle=\lambda^{K(q)} ; \lambda=L / l
$$

where " $<.>$ " indicates ensemble (statistical) averaging, $\varphi$ is the turbulent flux normalized such that $\left\langle\varphi_{\lambda}\right\rangle=1, K(q)$ is a convex function describing the scaling behaviour of the $q^{\text {th }}$ moment, $\lambda$ is the ratio of the (large) scale $L$, where the cascade starts, to the scale of observation $l$ (see Monin and Yaglom (1975) for an early discussion of cascades or Schertzer and Lovejoy (1987) for the "codimension multifractal formalism" used throughout this paper). In comparison, the quasi-Gaussian (nonintermittent) classical model is the (trivial) special case $K(q)=0$.

The usual "discrete in scale" model reproducing Eq. (1) is to consider a uniform (constant) large scale flux $\varphi_{0}(=1)$ which is iteratively divided into random substructures with the scale being reduced by integer ratios $\lambda_{0}$ at each step (usually $\lambda_{0}=2$ ). These smaller substructures multiplicatively modulate the larger scale field by independent identically distributed "multiplicative increments $\delta \varphi$ " so that after $n$ steps, at a given location in the space, the field $\varphi_{n}$ is given by $\varphi_{n}=\varphi_{0} \prod_{i=1}^{n} \delta \varphi_{i}$ while the overall scale range is $\lambda=\lambda_{o}^{n}$. If $\left\langle\delta \varphi^{q}\right\rangle=\lambda_{0}^{K(q)}$ we easily see that $\left\langle\varphi_{n}^{q}\right\rangle=\lambda^{K(q)}$. Since the cascade is multiplicative, its $\operatorname{logarithm} \Gamma_{n}=\log \varepsilon_{n}$, the "generator" is additive: $\Gamma_{n}=\Gamma_{0}+\sum_{i}^{n} \Delta \Gamma_{i}$ (with $\Delta \Gamma_{i}=\log \left(\delta \varepsilon_{i}\right)$ ). It is therefore not surprising that - due to the additive central limit theorem for the sums of identical independently distributed random variables - there exist specific (stable, attractive) "universal" forms for the exponent $K(q)$ :

$K(q)=\frac{C_{1}}{(\alpha-1)}\left(q^{\alpha}-q\right)$,

where $0 \leq C_{1} \leq d$ is the "codimension of the mean", which characterizes the sparseness of the set that gives the dominant contribution to the first order statistical moment (the mean), $d$ is the dimension of the space over which the cascade is observed (Schertzer and Lovejoy, 1987). The expression "dominant contribution" is an asymptotic result valid for large $\lambda$. In this limit there is an exact one to one correspondence between singularities $(\gamma)$ and statistical moments (Parisi and Frisch, 1985): $\gamma=K^{\prime}(q)$ so that the singularity corresponding to $q=1$ is $K^{\prime}(1)=C_{1}$. In addition, the same arguments show that the codimension (the difference of the dimension of space $d$ and the fractal dimension of this singularity) also equals $C_{1}$. The multifractal index $0 \leq \alpha \leq 2$ characterizes the degree of multifractality, i.e. the shape of the $K(q)$ function. It is also the Levy index of the generator. If the cascade is uni/mono- fractal, then $\alpha=0$, whereas $\alpha=2$ corresponds to the "lognormal" multifractal. A "universal multifractal" is the basin of attraction for wide variety of different multiplicative processes. For $\alpha<2$, Eq. (1b) is only valid for $q \geq 0$; the reason is that $\varepsilon$ is the exponential of an extremal Levy variable and when $\alpha<2$, the latter has diverging moments for all $q<0$. This means that the probability density of $\varepsilon$ has a logarithmic singularity for small $\varepsilon$ (except for $\alpha=2$, the Gaussian case).

In our analyses, we will see that the universal form (Eq. 1b) fits the empirical $K(q)$ quite well so that irrespective of whether the numerical models are indeed universal multifractals, the parameters $C_{1}, \alpha$ give very convenient parameterizations for their forms. Indeed, we have already seen that $C_{1}=K^{\prime}$ (1); we could similarly define $\alpha=K^{\prime \prime}(1) / K^{\prime}(1)$. For universal multifractals (Eq. 1b) this local $(q=1)$ characterization becomes global - i.e., is enough to describe the entire curve. In this way, the parameters $C_{1}$ and $\alpha$ still quantitatively characterize the statistics near the mean. In this paper, although we are primarily interested in establishing the basic predictions of multiplicative cascades (Eq. 1a), we also substantiate Eq. (1b) to some degree in Sect. 3.4, where we demonstrate a theoretically predicted "Levy collapse" of the moments. 
As we have seen from the brief history above, the hypothesis that high Reynolds number turbulence respects multiplicative cascades is physically based, so the paper aims to test the hypothesis on numerical simulations. While it might be possible that non-multiplicative processes may exist which satisfy Eq. (1a) (and perhaps even Eq. 1b); to our knowledge no such alternative models have been proposed.

\subsection{Estimating the turbulent fluxes}

In order to test Eq. (1a), we must therefore use an approach that does not require a priori assumptions about the physical nature of the relevant fluxes nor of their scale symmetries (isotropic or otherwise). If atmospheric dynamics are controlled by scale invariant turbulent cascades of various (scale by scale) conserved fluxes $\varphi$, then in a scaling regime, the fluctuations $\Delta f(\Delta x)$ in an observable $f$ (e.g. wind, temperature or radiance) over a distance $\Delta x$ are related to the turbulent fluxes by a relation of the form $\Delta f(\Delta x)=\varphi \Delta x^{H}$. This relation is a generalization of the classical laws of turbulence. For example, the Kolmogorov (1941) law for velocity fluctuations has $H=1 / 3$ and $\varphi=\varepsilon^{\eta}, \eta=1 / 3$ ( $\varepsilon$ is the energy flux), whereas the Corrsin-Obukhov law of passive scalar advection has $\varphi=\chi^{1 / 2} \varepsilon^{-1 / 6}$ where $\chi$ is the passive scalar variance flux (Corrsin, 1951 and Obukhov, 1949). Without knowing $\eta$ or $H$ - nor even the physical nature of the flux - we can use this to estimate the normalized (nondimensional) flux $\varphi$ ' at the smallest resolution of our data:

$\varphi^{\prime}=\varphi /\langle\varphi\rangle=\Delta f /\langle\Delta f\rangle$.

Note that if the fluxes are realizations of pure multiplicative cascades then the normalized $\eta$ power fluxes, $\varepsilon^{\eta} /\left\langle\varepsilon^{\eta}\right\rangle$, are also pure multiplicative cascades, so that $\varphi^{\prime}=\varepsilon^{\eta} /\left\langle\varepsilon^{\eta}\right\rangle$ is a normalized cascade quantity. The fluctuation $\Delta f(l)$, at small scales $\Delta x=l$ can be estimated in various ways; in 1-D a convenient method is to use absolute first differences: $\Delta f(l)=|f(x+l)-f(x)|$ or absolute second differences: $\Delta f(l)=|(f(x+l)+f(x-l)) / 2-f(x)|$. These "poor man's wavelets" are usually adequate - when as is typically the case $0 \leq H \leq 1$ or $0 \leq H \leq 2$ (first or second order differences, respectively) - but alternatively other definitions of fluctuations (other wavelets) could be used. In 2-D, convenient definitions of fluctuations (used below) are the (finite difference) Laplacian (estimated as the difference between the value at a grid point and the average of its neighbours: $\Delta f_{\Lambda}=\mid f(x, y)-(f(x+l, y)+f(x-l, y)+f(x, y+l)$ $+f(x, y-l)) / 4 \mid)$, or the modulus of a finite difference estimate of the gradient vector. The resulting high resolution flux estimates can then be degraded (by averaging) to lower resolutions.

Since empirical data are nearly always sampled at scales much larger than the dissipation scales, the above scaling range based technique has wide applicability. In numerical models however, where we have data down to the (model) dissipation range, we find that the approach still works but that the interpretation is a little different. To see this, consider the example of the energy flux $\varepsilon$, recalling that at the dissipation scale:

$\varepsilon \approx \nu \boldsymbol{v} \cdot \nabla^{2} \boldsymbol{v}$

where $v$ is the viscosity, $v$ the velocity (this is obtained from the Navier-Stokes equation by taking $\varepsilon=\partial v^{2} / \partial t$ and ignoring the dynamic terms which are unimportant for dissipation). Standard manipulations (e.g. Landau and Lifshitz, 1963) give:

$\varepsilon \approx v \sum_{i=1}^{3} \sum_{j=1}^{3}\left(\frac{\partial v_{i}}{\partial x_{j}}+\frac{\partial v_{j}}{\partial x_{i}}\right)^{2} \approx v\left(\frac{\Delta v}{\Delta x}\right)^{2}$

so that if $\Delta x$ is in the dissipation range (e.g. the finest resolution of the model) then:

$\Delta v \approx\left(\frac{\varepsilon}{v}\right)^{1 / 2} \Delta x$

The models considered here actually use hyper-viscosities, which have the advantage of confining the dissipation to a small range of scales (about a factor of 3 ). This means that their dissipation is due to a Laplacian raised to the power $h$ (typically $h$ is either 2 or 3) (e.g. Haugen and Brandenburg, 2004; Hamilton and Ohfuchi, 2007), we have:

$\varepsilon \approx v^{*} \boldsymbol{v} \cdot \nabla^{2 h} \boldsymbol{v}$

where $v^{*}$ is the hyperviscous coefficient chosen so that the field is indeed smooth when $\Delta x=l=1$ pixel. If $\Delta x$ is in the (smooth) dissipation regime, this leads to the estimate:

$\Delta v \approx\left(\frac{\varepsilon}{v^{*}}\right)^{1 / 2} \Delta x^{h}$

In all cases - irrespective of $h$ - for the normalized flux $\varphi$ ' we therefore have:

$\varphi^{\prime}=\frac{\varepsilon^{1 / 2}}{\left\langle\varepsilon^{1 / 2}\right\rangle}=\frac{\Delta v}{\langle\Delta v\rangle}$

We see that this is the same as Eq. (2), the only difference is that for the wind field, the dissipation exponent is $\eta=1 / 2$ rather than the value $\eta=1 / 3$ which holds in the scaling regime. If we introduce $K_{\eta}(q)$ which is the scaling exponent for the normalized $\eta$ flux $\varphi^{\prime}=\varepsilon^{\eta} /\left\langle\varepsilon^{\eta}\right\rangle$, (so that $\left.K_{1}(q)=K(q)\right)$ then taking $q^{\text {th }}$ moments of the latter, we obtain $K_{\eta}(q)=K_{1}(\eta q)-q K_{1}(\eta)$, which for universal multifractals (Eq. 1b) yields $K_{\eta}(q)=\eta^{\alpha} K_{1}(q)$ and $C_{1 \eta}=\eta^{\alpha} C_{1}$, so that comparing the dissipation estimate $(\eta=1 / 2)$ and the scaling range estimate $(\eta=1 / 3)$, we have:

$C_{1 \text { diss }}=\left(\frac{3}{2}\right)^{\alpha} C_{1 \text { scaling }}$

For the wind we find $\alpha \approx 1.8$ (see Table 2) so that $C_{1 \text { diss }} / C_{1 \text { scaling }} \approx 1.5^{1.8}=2.07$. 
Since passive scalars can be used as simplified models for the temperature and humidity fields which we analyze, the extension of this discussion to passive scalars is also relevant. It shows that the interpretation of the empirically/numerically estimated fluxes in terms of classical theoretical fluxes can be nontrivial. Denoting by $\rho$ the density of the passive scalar, and $\chi=\partial \rho^{2} / \partial t$ its variance flux, the dissipation range formula analogous to Eq. (3) is $\chi \approx \rho \kappa \nabla^{2} \rho$ ( $\kappa$ is the molecular diffusivity) leading to $\Delta \rho \approx(\chi / \kappa)^{1 / 2} \Delta x$ (with corresponding extensions to hyperviscous dissipation) whereas the corresponding formula in the scaling range is $\Delta \rho \approx \chi^{1 / 2} \varepsilon^{-1 / 6} \Delta x^{1 / 3}$ (Corrsin-Obukhov) which has the same dependency on $\chi$, but which also involves the energy flux; the combined effective flux $\phi \approx \chi^{1 / 2} \varepsilon^{-1 / 6}$ measured by the scaling method thus involves two (presumably statistically dependent) cascade quantities. In summary, although both dissipation and scaling ranges can be used to test for multiplicative cascades and to quantify their variability, the relation between the two is not necessarily trivial.

A final practical consideration is that in the analyses, the outer scale is not known a priori, but is an empirically estimated parameter. It is therefore convenient to define a reference scale $L_{\text {ref }}$ so that $\lambda=L_{\text {ref }} / L$. If the cascade starts at the "effective outer scale" $L_{\text {eff }}$ then the corresponding ratio is $\lambda_{\text {eff }}=L_{\text {ref }} / L_{\text {eff }}$ and the normalized moments $M_{q}=\left\langle\varphi_{\lambda}^{q}\right\rangle /\left\langle\varphi_{1}\right\rangle^{q}$ are expected to obey the generic multiscaling relation:

$M_{q}=\left(\frac{\lambda}{\lambda_{\text {eff }}}\right)^{K(q)} ; \lambda=L_{\mathrm{ref}} / L ; \lambda_{\mathrm{eff}}=L_{\mathrm{ref}} / L_{\mathrm{eff}}$

where " $<$.>" indicates statistical (ensemble) averaging and $L_{\text {eff }}$ is the effective outer scale of the cascade. $\left\langle\varphi_{1}\right\rangle$ is the ensemble mean large scale (i.e. the climatological value). $\lambda$ is a convenient scale ratio based on the largest great circle distance on the earth: $L_{\text {ref }}=L_{\text {earth }}=20000 \mathrm{~km}$ and the scale ratio $\lambda / \lambda_{\text {eff }}$ is the overall ratio from the scale where the cascade started to the intermediate scale $L_{\mathrm{ref}} \geq L \geq l$.

\section{Analysis of models}

\subsection{Discussion}

To our knowledge there have been no attempts to directly check Eq. (1a) on either DNS or geophysical numerical simulations. The closest is perhaps the multifractal characterization of time signals in turbulent shell models (Biferale, 2003) or in "scaling cascade of gyroscopes" models (Chigirinskaya and Schertzer, 1996; Chigirinskaya et al., 1998). While the former discretizes the NS equations in Fourier space keeping a small and fixed number of degrees of freedom per octave in scale, the latter more realistically discretizes the equations on a dyadic tree structure such that the number of degrees of freedom increases with wavenumber. While the former approach leads to multifractal behaviour in time (and hence presumably temporal cascades), the latter leads to space-time cascades and are hence particularly relevant here (interestingly, they yield comparable universal multifractal parameters). Other relevant connections between the cascade prediction, Eq. (1a), and dynamical equations are the studies of temporal scaling (Syroka and Toumi, 2001; Blender and Fraedrich, 2003; Fraedrich and Blender, 2003), and temporal multifractality of climate models (Royer et al., 2008). The companion papers Stolle (2009) and Stolle et al. (2009) extends the present analyses to the time domain and makes systematic space-time comparisons.

Our primary goal is to check the basic prediction of the multiplicative cascade models (Eq. 1b) directly on simulations of the atmosphere (both forecasts and reanalyses). This choice was made both due to the ready availability of large numbers of realizations and due to the scientific (weather, climate) significance of the results. Furthermore, as mentioned previously, we expected to have a significant range of scales exhibiting cascade behaviour because hyper-viscosity restricts most the effects of dissipation to a narrow range of scales (see however Frisch et al. (2008) for possible "side effects" of using hyperviscosity).

Over the scaling/inertial range, there are three main differences between the cascade structure of 3-D DNS and of atmospheric models. First, in the former there is a single (energy flux) cascade, while in the latter we expect there to be several coupled cascades. The second is that due to gravity, the atmosphere is stratified so that the cascades are anisotropic (Schertzer and Lovejoy, 1987); indeed as mentioned earlier - due to the $10 \mathrm{~km}$ scale height of the mean pressure field, isotropic models require at least two cascade regimes for each flux. The third is that in DNS applications to fully developed turbulence the forcing is deliberately confined to the largest scales and the dissipation to the smallest scale. The cascade thus occurs in a source and sink free "inertial range". In comparison, the atmospheric boundary conditions are quite different. In particular both the topography (Gagnon et al., 2006) and the critical energy-containing short and long wavelengths radiances responsible for the forcing (Lovejoy et al., 2001, 2009a) have been found to have wide scale range cascade structures so that the boundary conditions and flux sources and sinks apparently do not introduce characteristic scales and so need not destroy the cascades.

\subsection{The model outputs}

\subsubsection{Discussion}

We chose two forecast models and one reanalysis, all recognized as being state-of-the-art: the Canadian Meteorological Centre (CMC) Global Environmental Multiscale (GEM) model, the NOAA Global Forecast System (GFS) model and the European Centre for Medium range Weather Forecasting's (ECMWF) reanalysis (ERA40). For the products of all three models, we analyzed the three most dynamically significant fields: temperature $(T)$, east-west $(u)$ wind fields, the 
specific humidity $\left(h_{s}\right.$; GEM, ERA 40$)$ and the relative humidity $\left(h_{r}, \mathrm{GFS}\right)$. In order to check for possibly latitude dependencies, analyses were made both in the regions between $\pm 30^{\circ}$ (tropics) and $\pm 45^{\circ}$ latitude. We did not consider the spatial cascade structure of climate models since their spatial resolutions are relatively low. Table 1 shows the main model characteristics relevant to the analyses here.

\subsubsection{The Canadian Meteorological Centre (CMC) Global Environmental Multiscale (GEM) model}

This model is on a $0.25^{\circ} \times 0.3^{\circ}$ horizontal grid with 28 levels and our analysis used a $0.6^{\circ} \times 0.6^{\circ}$ resolution product (about $66 \mathrm{~km}$ resolution - the high-resolution CMC GRIB dataset). We used 505 realizations at $12 \mathrm{~h}$ intervals taken from 20 September 2007 to 2 June 2008 which are initialized at either $12 \mathrm{Z}$ or $00 \mathrm{Z}$ and analysed the initial objective analysis, the $48 \mathrm{~h}$ forecast, and a 144 hour forecast. Days outside of this time interval were not used because the model resolution and grid were changed and assimilating results outside of this timespan to the results given here is non-trivial. 4DVAR 6-hourly assimilation is used (CMC Global Data Assimilation System, DAS). The model can be adapted; see Côté et al. (1998a, b) for more details.

\subsubsection{The NOAA Global Forecast System (GFS) model}

As with the CMC GEM model, the GFS is a global NWP model, which we also analyzed at its analysis and $48 \mathrm{~h}$ forecast. It uses T254 Spectral and $768 \times 384$ Gaussian grids on 64 vertical levels. The data is obtained on a $1^{\circ} \times 1^{\circ}$ resolution grid every $6 \mathrm{~h}$; each initialization starts at $00 \mathrm{Z}, 06 \mathrm{Z}$, $12 Z$, or $18 Z$. The data were taken from 1 August 2007 to 30 June 2008 (with the exception of $700 \mathrm{mb} u$, where the first 61 days were corrupted). The assimilation system used is 3DVAR (Okamoto and Derber, 2006) with an assimilation cycle of $6 \mathrm{~h}$. A total of 1340 realizations were analyzed, every $6 \mathrm{~h}$. For more information, see Sela $(1982,1988)$ and NCEP office note 442 (2003).

\subsubsection{The European Centre for Medium range Weather Forecasting's (ECMWF) reanalysis (ERA40) product}

A reanalysis is the result of assimilating atmospheric measurements with numerical forecasts in an attempt to obtain realistic fields; it is a very model dependent "product". Here a 6-hourly 3DVAR assimilation cycle was used. For ERA40, the dynamic variables are on a 2-D triangular spherical harmonic truncation T159 with 60 levels (Uppala et al., 2005) projected onto a $1^{\circ} \times 1^{\circ}$ resolution grid and interpolated to constant pressure levels $(1000 \mathrm{mb}, 850 \mathrm{mb}, 700 \mathrm{mb}, 500 \mathrm{mb}$, $200 \mathrm{mb}$ ). We analyzed the most recent three years of the reanalysis: September 1999-August 2002 with a total of 4380 realizations analyzed (every $6 \mathrm{~h}$ ). See Uppala et al. (2005) for more details.

\subsection{Testing multiplicative cascades}

Figure 1 shows plots of $\log _{10} M_{q}$ for $T, u, h_{s}$ for both the GEM analysis ( $t=0$; Fig. $1 \mathrm{a}, \mathrm{c}, \mathrm{e})$ and the $t=144 \mathrm{~h}$ forecast (Fig. 1b, d, f) at $1000 \mathrm{mb}$, while Fig. 2 shows the corresponding plots $\left(h_{r}\right.$ instead of $h_{s}$ for GFS) for ERA40 (Fig. 2a, c, e) and GFS analysis datasets (Fig. 2b, d, f) at $1000 \mathrm{mb}$ and Fig. 3 shows the moments of $u$ field at $700 \mathrm{mb}$ for all datasets taken between $\pm 30^{\circ}$ (Fig. 3a, c, e) and $\pm 45^{\circ}$ latitude (Fig. 3b, d, f). Note that the $1000 \mathrm{mb}$ fields are more influenced by the data - although with nontrivial effects where the topography is important - while the $700 \mathrm{mb}$ fields are more representative of the free atmosphere. All the plots display the typical cascade "signature" - the converging straight lines predicted by Eq. (10). The regressions were performed by minimizing the deviations (Eq. 11, defined below) through a common intersection point over the range from the grid scale to $5000 \mathrm{~km}$. Since we test the predictions of multiplicative cascade models, all lines were forced to pass through the same point (Eq. 10 with $\lambda=\lambda_{\text {eff }}$ ). Two remarkable features are: a) the cascades begin at an outer scale very close to the scale of the planet and b) up to $\approx 5000 \mathrm{~km}$ the cascade structure is accurately followed. Note that $L_{\text {eff }}>L_{\text {ref }}$ $(=20000 \mathrm{~km})$ simply indicates that there is residual variability at planetary scales, while $L_{\text {eff }}<L_{\text {ref }}$ indicates that it requires a certain range of scales for the scaling to become developed.

To quantify the accuracy with which Eq. (10) is satisfied, we characterize the deviations using the mean absolute residuals for the statistical moments $M_{q}$ of order $q$ from 0.0 to 2.0, for all points between the scale of the grid and $5000 \mathrm{~km}$ :

$\Delta=\overline{\left|\log _{10}\left(M_{q}\right)-K(q) \log _{10}\left(\lambda / \lambda_{\text {eff }}\right)\right|}$.

To convert $\Delta$ to a percent deviation, $\delta=100\left(10^{\Delta}-1\right)$ was used; we found $\delta< \pm 2 \%$ for all analyzed fields and $\delta< \pm 1 \%$ for all initial analyses and short-range forecasts. This accuracy is very close to those of the same moments of the visible and IR radiances over the range $10-5000 \mathrm{~km}(\approx \pm 0.5 \%$, Lovejoy et al., 2009a).

For the fields, the scaling extends from the grid size up to $\sim 5000-20000 \mathrm{~km}$. It is worth noting that the outer scales for these fields $(\sim 8000-27000 \mathrm{~km})$ are approximately the same as the outer scales of the radiances $(\sim 5000-32000 \mathrm{~km}$ in Lovejoy et al., 2009a). We also mention that the $C_{1}$ 's are not too different from the $C_{1}$ 's for passive scalars $(\sim 0.1)$ (Lilley et al., 2004). Similar results were found for the moments of the 2-D wind estimate of the energy flux.

From Table 2, we see that very similar results were found for GEM forecasts (Fig. 1b, d, f) and the GFS model; for example, the deviations are of the order $\pm 0.3 \%$ for the GEM and $\pm 0.5 \%$ for the $48 \mathrm{~h}$ GFS forecast for the analysis and $48 \mathrm{~h}$ forecast (Table $2 \mathrm{a}, \mathrm{b}, \mathrm{c}$ ). These small deviations allow us to conclude that the analyses and models do indeed accurately have a cascade structure. Overall, from the table, 

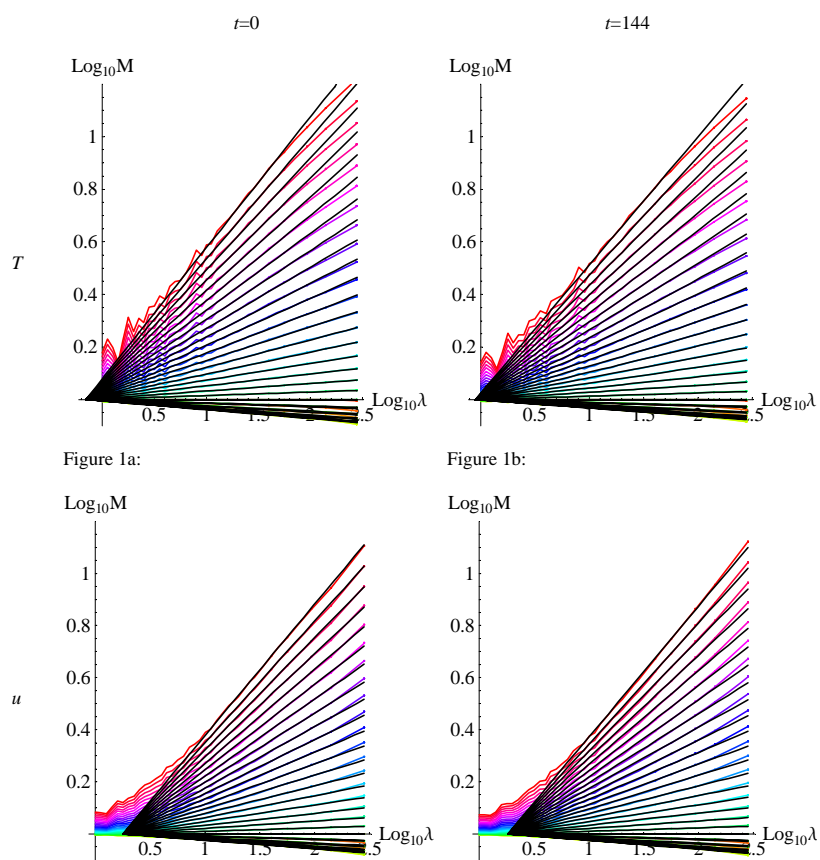

Figure 1b:
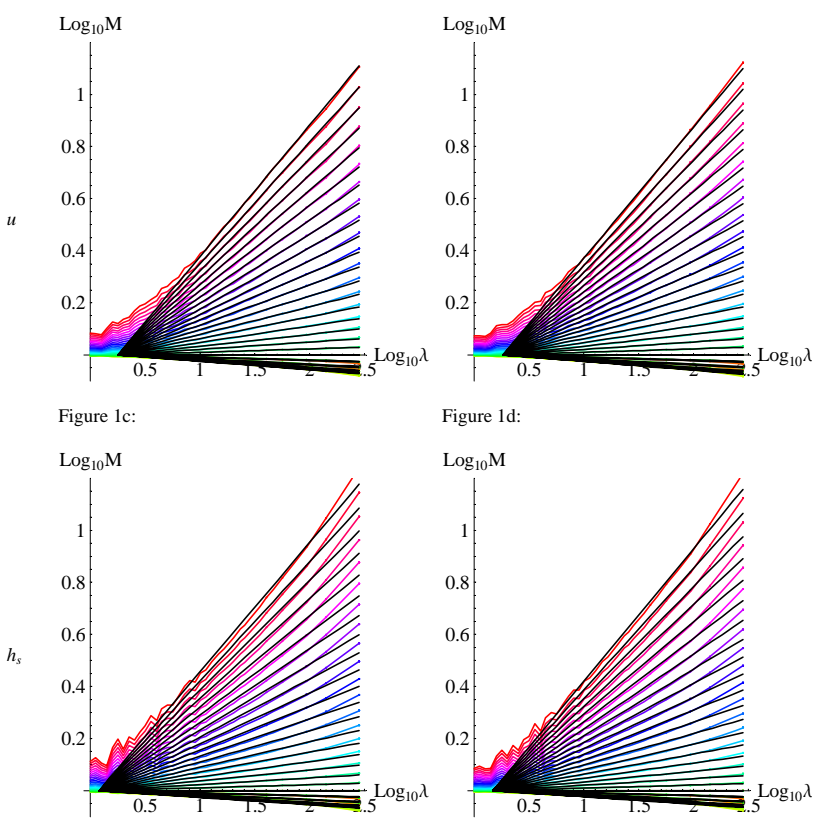

Figure 1e:

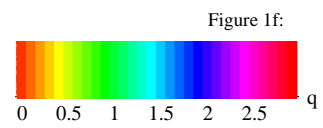

Fig. 1. Moments of fields for GEM at $1000 \mathrm{mb}$ for $q=0.0$ to 2.9 $\left(q>1.0: \log _{10} M_{q}>0\right.$, monotonically increasing; $q<1.0: \log _{10}$ $\left.M_{q}<0\right)$ in steps of $0.1, \lambda=L_{\text {earth }} / L, L_{\text {earth }}=20000 \mathrm{~km}$. The $q^{t h}$ moment colour key is given at the bottom of the figure $(q=0$ (reddish-orange) to $q=2.9$ (red)). Left, at time $t=0$, on the right, the $144 \mathrm{~h}$ forecast; from top to bottom, temperature, east-west wind, specific humidity, all between $\pm 30^{\circ}$ latitude. (a) temperature at initialization; (b) temperature at $144 h$ forecast; (c) $u$ wind at initialization; (d) $u$ wind at $144 \mathrm{~h}$ forecast; (e) $h_{s}$ at initialization; (f) $h_{s}$ at $144 \mathrm{~h}$ forecast. For the parameters, refer to Table $2 \mathrm{~b}$.

we can also see that the $K(q)$ "shape parameter" - the difficult to estimate multifractal index $\alpha-$ is roughly constant at $\alpha \approx 1.8 \pm 0.1$. We examine the issue of the accuracy of this parametric representation, Eq. (1b), in Sect. 3.4. From Table $2 \mathrm{a}$, we see that the scale by scale characterization of the intermittency near the mean $\left(C_{1}\right)$ has a tendency to decrease with altitude, this being somewhat amplified by a decrease in the external scale (which decreases all the moments by the same factor). Interestingly, the $C_{1}$ values are very similar for the different fields (it is slightly larger for the humidity), although significantly, the $C_{1}$ are quite a bit larger than those measured by aircraft (Sect. 2.5 in Lovejoy et al., 2009b), also shown in the table.

Also in Table $2 \mathrm{a}$ is a comparison of aircraft estimates of the parameters from Lovejoy et al. (2009b) which included an in-depth evaluation of the optimum scale range $(4-40 \mathrm{~km})$ needed to avoid spurious aircraft effects. Since the aircraft fluxes were estimated in the scaling regime, we don't expect the parameters to be identical to the dissipation range fluxes estimated here (see the discussion in Sect. 2). However, when the theoretical correction factor (assuming $\alpha=1.8$ ) is used, the agreement is seen to be good (it is much improved). Note that the agreement is not expected to be perfect since Eq. (9) depends on an explicit identification of the conserved flux; while the argument is fairly robust for the velocity - as indicated - it is not so for the other quantities. In addition both the aircraft and model estimates will have some systematic biases so that our main point is that the results are plausibly consistent.

In order to estimate the parameter uncertainties, we calculated them over subsets of the data for $\pm 30^{\circ}$ - each subset is about 1 month long - as indicated in the last row of Table 1. For all datasets, the maximum and minimum value of $\alpha$ for each subset in every dataset differ by less than 0.1 with the following exceptions: ERA40 $h_{s} 850 \mathrm{mbar}(0.27)$, ERA40 $h_{s} 200 \mathrm{mbar}(0.22)$. The maximum and minimum values of $C_{1}$ for each subset differ by less than 0.008 for GEM and GFS and less than 0.014 for ERA40. The maximum variation in the estimates of $\log _{10} \lambda_{\text {eff }}$ is less than 0.08 for GEM and GFS and less than 0.2 for ERA40 with the following exceptions for ERA40: ( $u 850$ mbar (0.2), T 200 mbar (0.2), $h_{s} 850$ mbar (0.3), $\left.h_{s} 200 \mathrm{mbar}(0.5)\right)$. For the most part, the deviations from the parameters estimated from 1 year of data are small, but it should not be surprising that there are occasional larger deviations because large amounts of data are needed to estimate parameters accurately for multifractal data.

In Table $2 \mathrm{~b}$, we compare the two forecast models (GEM, GFS) in order to see if there are any systematic trends as the model integration times increase so that the effect of initial conditions becomes less and less important. No systematic trends are obvious, although for the $144 \mathrm{~h}$ GEM forecast, the scaling is a bit poorer (although it is still very good with deviations less than $\pm 1.6 \%$ ). The scaling of the longest available forecast is important since it is of interest to determine whether the cascade structure is imposed by the analysis, or whether it is generated intrinsically by the model (or more likely a combination of both but with possibly nonidentical cascades). Since even after $144 \mathrm{~h}$ the initial conditions have not been completely "forgotten" these results only support the hypothesis that the long time behaviour of the model is cascade-like, they do not fully establish it. 
Table 1. Comparison of various model parameters. The time step is the model integration time step. The last row indicates the size of the subsets used to estimate the uncertainties (see the text).

\begin{tabular}{llll}
\hline Model & GEM & GFS & ERA 40 \\
\hline $\begin{array}{l}\text { Time step } \\
\text { (minutes) } T_{s t}\end{array}$ & 22.5 & 7.5 & 30 \\
$\begin{array}{l}\text { Model Spatial } \\
\text { resolution (grid size) }\end{array}$ & $0.25^{\circ} \times 0.3^{\circ}$ & $0.47^{\circ} \times 0.47^{\circ}$ & $1.125^{\circ} \times 1.125^{\circ}$ \\
$\begin{array}{l}\text { Spatial resolution of } \\
\text { the analysis } L_{i}\end{array}$ & $0.6^{\circ} \times 0.6^{\circ}$ & $1^{\circ} \times 1^{\circ}$ & $1^{\circ} \times 1^{\circ}$ \\
$\begin{array}{l}\text { Number of vertical } \\
\text { levels }\end{array}$ & 28 & 64 & 60 \\
$\begin{array}{l}\text { Number of realizations } \\
\text { in the sample }\end{array}$ & 505 & 1340 & 4384 \\
$\begin{array}{l}\text { Time interval between } \\
\text { realizations (hours) }\end{array}$ & 12 & 6 & 6 \\
$\begin{array}{l}\text { Size of dataset } \\
\text { subset }\end{array}$ & $25-25.5$ days & $30-30.5$ days & 1 month \\
\hline
\end{tabular}

Table 2a. Intercomparison of cascade parameters for the initial $(t=0)$ fields for various fields at $1000,700 \mathrm{mb}$. The triplets of values are for, ERA40 (denoted by "ERA"), GEM, GFS respectively. The aircraft estimates are from about $200 \mathrm{mb}$ (the figure in parentheses is from aircraft analyses (Lovejoy et al., 2009c, Table 3 for $C_{1}, \alpha$, Table 1 for $L_{\text {eff }}, \delta$ ), the second is corrected by the factor (3/2) ${ }^{\alpha}$ needed - at least for the wind field - to estimate the dissipation scale $C_{1}$ from the scaling range $C_{1}$, see Eq. 9).

\begin{tabular}{|c|c|c|c|c|c|c|c|c|c|c|c|c|}
\hline & \multicolumn{3}{|c|}{$C_{1}$} & \multicolumn{3}{|c|}{$\alpha$} & \multicolumn{3}{|c|}{$L_{\text {eff }}(\mathrm{km})$} & \multicolumn{3}{|c|}{$\delta(\%)$} \\
\hline & ERA & GEM & GFS & ERA & GEM & GFS & ERA & GEM & GFS & ERA & GEM & GFS \\
\hline$T(1000)$ & 0.113 & 0.125 & 0.142 & 1.94 & 1.64 & 1.72 & 21900 & 25800 & 28000 & 0.31 & 0.27 & 0.59 \\
\hline$T(70$ & 0.094 & 0.077 & 0.080 & 2.11 & 1.94 & 2.00 & 14500 & 8300 & 8600 & 0.29 & 0.47 & 1.02 \\
\hline$T(200)$ & 0.080 & 0.080 & 0.065 & 1.93 & 1.88 & 1.85 & 12100 & 10700 & 7800 & 0.30 & 0.36 & 1.17 \\
\hline$T$ (aircraft) & \multicolumn{3}{|c|}{$(0.052), 0.107$} & \multicolumn{3}{|c|}{1.78} & \multicolumn{3}{|c|}{5000} & \multicolumn{3}{|c|}{0.5} \\
\hline$u(1000)$ & 0.105 & 0.121 & 0.114 & 1.93 & 1.68 & 1.80 & 12900 & 11000 & 12300 & 0.33 & 0.32 & 0.54 \\
\hline$u(7$ & 0.096 & 0.104 & 0.08 & 1.9 & 1.86 & $1.8^{\circ}$ & 12000 & 11000 & 9000 & 0.24 & 0.29 & 0.83 \\
\hline$u(200)$ & 0.075 & 0.085 & 0.073 & 1.92 & 1.85 & 1.89 & 15900 & 16300 & 9000 & 0.267 & 0.35 & 0.76 \\
\hline$u$ (aircraft) & \multicolumn{3}{|c|}{$(0.040), 0.088$} & \multicolumn{3}{|c|}{1.94} & \multicolumn{3}{|c|}{25000} & \multicolumn{3}{|c|}{0.8} \\
\hline$h_{s}, h_{r}(1000)$ & 0.121 & 0.109 & 0.128 & 2.03 & 1.81 & 1.86 & 19800 & 15900 & 21700 & 0.33 & 0.51 & 0.46 \\
\hline$h_{s}, h_{r}(700)$ & 0.094 & 0.100 & 0.091 & 1.75 & 1.60 & 1.74 & 11000 & 11800 & 9000 & 0.26 & 0.37 & 0.46 \\
\hline$h_{s}, h_{r}(200)$ & 0.085 & 0.109 & 0.100 & 1.73 & 1.54 & 1.70 & 50000 & 33000 & 9700 & 0.47 & 0.56 & 0.64 \\
\hline$h$ (aircraft) & \multicolumn{3}{|c|}{$(0.040), 0.083$} & \multicolumn{3}{|c|}{1.81} & \multicolumn{3}{|c|}{10000} & \multicolumn{3}{|c|}{0.5} \\
\hline
\end{tabular}

In Table 2c, we compare the cascade parameters calculated between $\pm 30^{\circ}$ and $\pm 45^{\circ}$ in an admittedly crude attempt to see if there are any detectable differences between the tropics and a region including the midlatitudes. A latitude effect might arise because of the importance of the Coriolis force in the midlatitudes which makes the large scale winds quasigeostrophic. We find that the only notable difference is that the outer scale is systematically larger for the $\pm 45^{\circ}$ region. It could be noted that although we did not use equal area grids, the variation in grid size is still fairly small even at $45^{\circ}$.

\subsection{Universality and Levy collapse}

The main aim of this paper is to establish the fundamental prediction of multiplicative cascade models, Eq. (1a). In Table 2 a-c we gave estimates of the external scale ( $\left.\lambda_{\text {eff }}, L_{\text {eff }}\right)$ and the relative deviation of the model outputs from the theoretical predictions, averaging over the scale range $5000 \mathrm{~km}$ down to the grid scale and over the statistical moments $q \leq 2$. Up until now, the additional hypothesis - that the outputs belong to multifractal universality classes - was only used to 
Table 2b. An intercomparison of the $1000 \mathrm{mb}$ fields, the triplets representing the parameter estimates for integrations of $t=0,48,144 \mathrm{~h}$.

\begin{tabular}{|c|c|c|c|c|c|c|c|c|c|c|c|c|}
\hline & \multicolumn{3}{|c|}{$C_{1}$} & \multicolumn{3}{|c|}{$\alpha$} & \multicolumn{3}{|c|}{$L_{\mathrm{eff}}(\mathrm{km})$} & \multicolumn{3}{|c|}{$\delta(\%)$} \\
\hline & $0 \mathrm{~h}$ & $48 \mathrm{~h}$ & $144 \mathrm{~h}$ & $0 \mathrm{~h}$ & $48 \mathrm{~h}$ & $144 \mathrm{~h}$ & $0 \mathrm{~h}$ & $48 \mathrm{~h}$ & $144 \mathrm{vh}$ & $0 \mathrm{~h}$ & $48 \mathrm{~h}$ & $144 \mathrm{~h}$ \\
\hline$T(\mathrm{GEM})$ & 0.125 & 0.115 & 0.113 & 1.64 & 1.68 & 1.69 & 25700 & 20500 & 21000 & 0.27 & 0.26 & 0.67 \\
\hline$T$ (GFS) & 0.142 & 0.138 & & 1.72 & 1.71 & & 27900 & 26000 & & 0.59 & 0.60 & \\
\hline$u(\mathrm{GEM})$ & 0.121 & 0.122 & 0.122 & 1.68 & 1.62 & 1.63 & 11000 & 11000 & 11000 & 0.32 & 0.36 & 1.14 \\
\hline$u$ (GFS) & 0.114 & 0.107 & & 1.80 & 1.84 & & 12300 & 11200 & & 0.54 & 0.64 & \\
\hline$h_{S}(\mathrm{GEM})$ & 0.109 & 0.106 & 0.107 & 1.81 & 1.80 & 1.80 & 15900 & 13800 & 13500 & 0.51 & 0.49 & 1.54 \\
\hline$h_{r}(\mathrm{GFS})$ & 0.128 & 0.128 & & 1.86 & 1.81 & & 21700 & 20900 & & 0.46 & 0.46 & \\
\hline
\end{tabular}

Table 2c. An intercomparison of ranges of values of data in $700 \mathrm{mb}$ fields between, pairs representing parameter estimates at analysis time step for $\pm 30^{\circ}$ and $\pm 45^{\circ}$. * The first two months were excluded because of corrupt data.

\begin{tabular}{|c|c|c|c|c|c|c|c|c|}
\hline & \multicolumn{2}{|c|}{$C_{1}$} & \multicolumn{2}{|c|}{$\alpha$} & \multicolumn{2}{|c|}{$L_{\mathrm{eff}}(\mathrm{km})$} & \multicolumn{2}{|c|}{$\delta(\%)$} \\
\hline & $\pm 30^{\circ}$ & $\pm 45^{\circ}$ & $\pm 30^{\circ}$ & $\pm 45^{\circ}$ & $\pm 30^{\circ}$ & $\pm 45^{\circ}$ & $\pm 30^{\circ}$ & $\pm 45^{\circ}$ \\
\hline$T$ (ERA40) & 0.094 & 0.091 & 2.11 & 2.11 & 14500 & 21400 & 0.288 & 0.274 \\
\hline$T$ (GEM) & 0.077 & 0.082 & 1.94 & 2.19 & 8300 & 17000 & 0.47 & 0.36 \\
\hline$T$ (GFS) & 0.080 & 0.084 & 2.00 & 2.03 & 8600 & 11100 & 1.03 & 0.88 \\
\hline$u$ (ERA40) & 0.096 & 0.094 & 1.93 & 1.90 & 12000 & 14000 & 0.239 & 0.225 \\
\hline$u(\mathrm{GEM})$ & 0.104 & 0.106 & 1.86 & 1.84 & 10900 & 12600 & 0.295 & 0.34 \\
\hline$u(\mathrm{GFS})^{*}$ & 0.085 & 0.082 & 1.87 & 1.87 & 9000 & 10100 & 0.83 & 0.69 \\
\hline$h_{s}($ ERA40) & 0.093 & 0.097 & 1.74 & 1.73 & 11000 & 14300 & 0.259 & 0.224 \\
\hline$h_{s}(\mathrm{GEM})$ & 0.100 & 0.105 & 1.60 & 1.61 & 11800 & 14800 & 0.37 & 0.33 \\
\hline$h_{r}(\mathrm{GFS})$ & 0.091 & 0.094 & 1.74 & 1.72 & 9000 & 9000 & 0.46 & 0.39 \\
\hline
\end{tabular}

justify a two parameter $\left(C_{1}, \alpha\right)$ regression. Since both parameters have fairly simple interpretations (in terms of the closest monofractal approximation $\left(C_{1}\right)$ and the curvature of $K(q)$ near $q=1(\alpha))$, the variation of these parameters with model type, integration time etc. can conveniently be used to characterize the variation of the cascade structure. However, we also argued that there are basic physical, mathematical reasons (essentially the existence of a kind of multiplicative central limit theorem) that make it plausible that the model outputs fall into special universality classes in which the basic scale invariant exponent $K(q)$ is given by Eq. (1b) characterized by just two parameters $C_{1}, \alpha$. In this section we explore the accuracy of Eq. (1b).

We must first note that even for perfect universal multifractal processes, Eq. (1b) is only expected to be strictly valid for the "bare" cascade properties, i.e. those of an infinite ensemble of realizations of a cascade developed down to scale $L=L_{\text {ref }} / \lambda$ and then stopped. However, when analyzing the model output, we analyzed the "dressed" quantities, i.e. those obtained by degrading (by integrating) the resolution of a cascade developed to small scales (for the "bare"/"dressed" distinction see Schertzer and Lovejoy, 1987), and these were estimated using only a finite number of realizations. Both the bare/dressed distinction and the finite sample size give rise to (first or second order) "multifractal phase transitions" (Schertzer et al., 1993). This means that the measured (dressed) moments will only have the theoretical bare exponent $K(q)$ for $q$ below a critical moment $q_{c}$ beyond which there is a - multifractal phase transition - where $K$ becomes asymptotically linear (a sample size-dependent effect corresponding to the domination of the statistics by the largest flux values present). In Fig. 4, using some representative comparisons of $K(q)$ and the fits to the universal multifractal form (Eq. 1b), we see that the universal form is in fact very closely followed except for hints of linearity for some $q \geq q_{c}>2$. In fact for $q<2$, the deviations from the universal form are of the order $\pm 1- \pm 2 \%$.

Assuming that $q_{c}>2$ then, the universal form Eq. (1b) should hold over the range $0<q<2$ (we do not consider $q<0$ since on the one hand the statistics are very sensitive to very small gradients and are hence unreliable, and on the other, for universal multifractals with $\alpha<2$, they should diverge anyway). In this case, one can attempt to "collapse" the log moments $\log M_{q}$ to a unique curve by dividing $\log M_{q}$ by the 

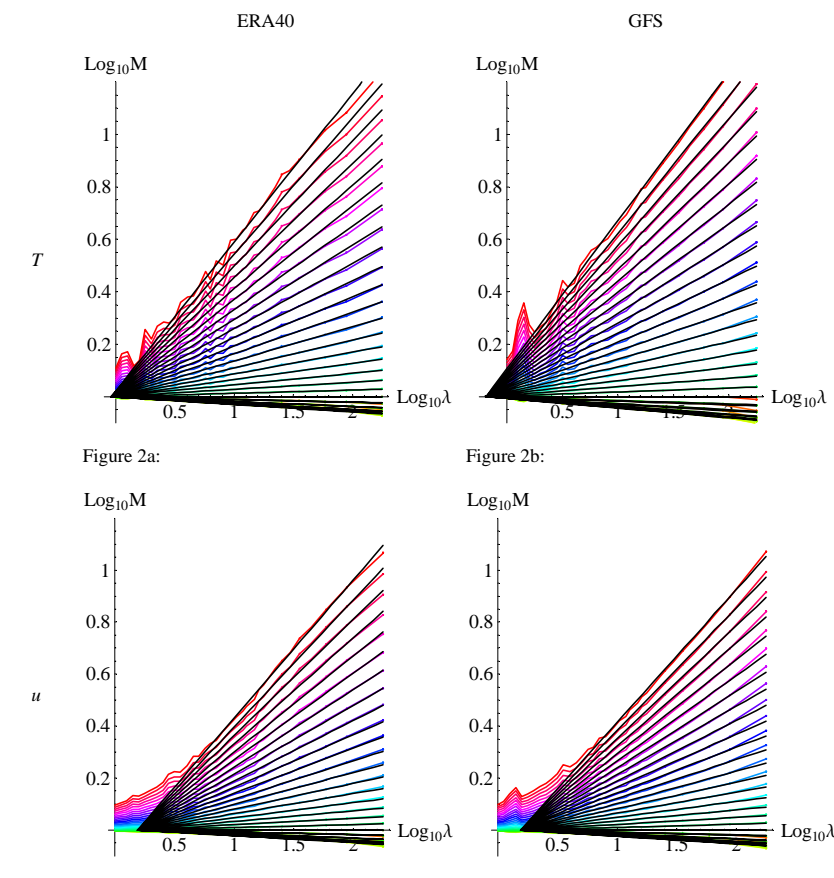

Figure 2b:
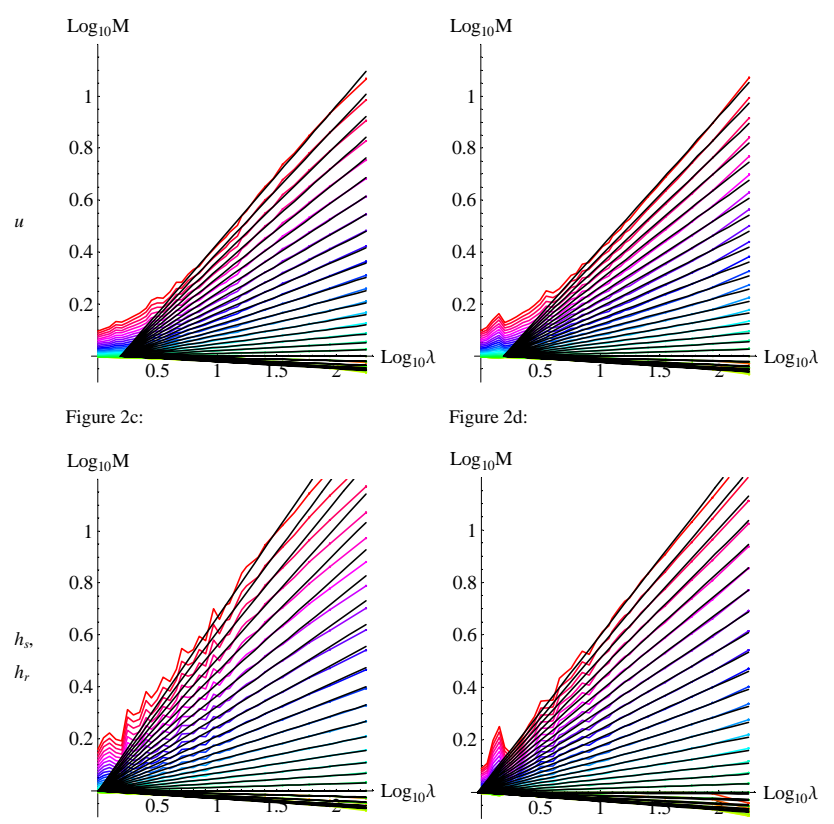

Figure 2d:

Figure 2e:

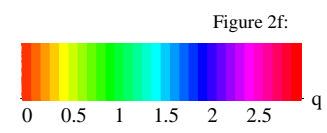

Fig. 2. Moments of the ERA40, GFS $(t=0)$ fields at $1000 \mathrm{mbar}$ for $q=0.0$ to $2.9 \quad\left(q>1.0: \quad \log _{10} M_{q}>0\right.$, monotonically increasing; $\left.q<1.0: \log _{10} M_{q}<0\right)$ in steps of $0.1, \lambda=L_{\text {earth }} / L$, $L_{\text {earth }}=20000 \mathrm{~km}$. The $q^{\text {th }}$-moment colour key is given at the bottom of the figure ( $q=0$ (reddish-orange) to $q=2.9$ (red)). On the left, ERA 40, on the right, the GFS, top to bottom: temperature, east-west wind, and humidity, between $\pm 30^{\circ}$ latitude. (a) ERA40 temperature; (b) GFS analysis temperature; (c) ERA40 $u$ wind; (d) GFS analysis $u$ wind; (e) ERA40 $h_{s}$; (f) GFS analysis $h_{r}$. For the corresponding parameters, refer to Table $2 \mathrm{a}$.

theoretical $K(q)$ for (say) $C_{1}=1$, i.e. by dividing by $\left(q^{\alpha}-q\right) /(\alpha-1)$. If $M_{q}$ does indeed follow Eq. (1a) and (1b) with parameters $C_{1}, \alpha$, then can define the "collapsed moments":

$M_{q}^{\prime}=\left(M_{q}\right)^{\frac{(\alpha-1)}{q^{\alpha}-q}}$
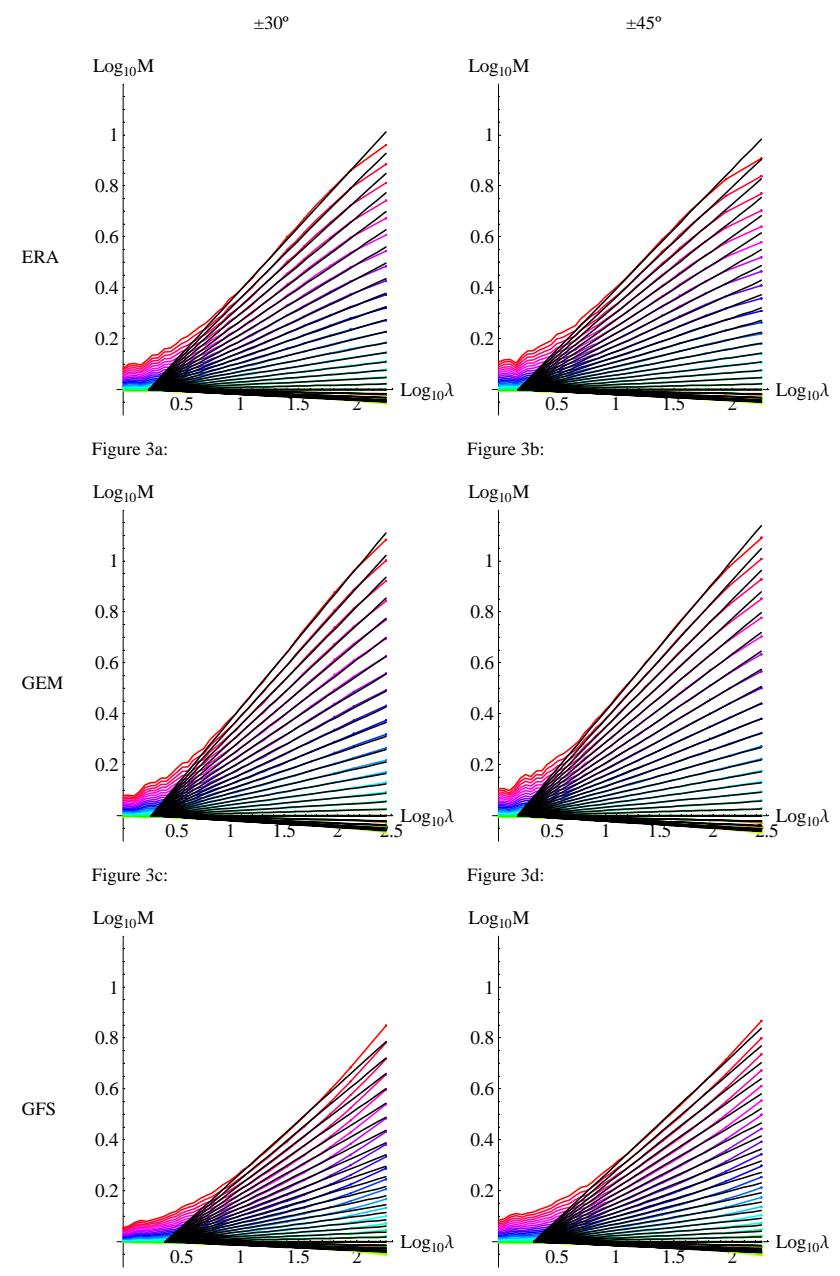

Figure 3e:

Figure 3f:

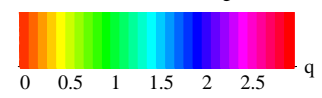

Fig. 3. Moments of $u$ fields analysis time step at 700 mbar for $q=0.0$ to 2.9 ( $q>1.0$ : $\log _{10} M_{q}>0$, monotonically increasing; $q<1.0$ : $\left.\log _{10} M_{q}<0\right)$ in steps of $0.1, \lambda=L_{\text {earth }} / L, L_{\text {earth }}=20000 \mathrm{~km}$. The $q^{t h}$-moment colour key is given at the bottom of the figure $(q=0$ (reddish-orange) to $q=2.9$ (red)). The left column are analyses between $\pm 30^{\circ}$ latitude, the right hand between $\pm 45^{\circ}$, top to bottom, ERA 40 (denoted by "ERA"), GEM $(t=0)$, GFS $(t=0)$. (a) ERA40 between $\pm 30^{\circ}$ latitude; (b) ERA40 between $\pm 45^{\circ}$ latitude; (c) GEM between $\pm 30^{\circ}$ latitude; (d) GEM between $\pm 45^{\circ}$ latitude; (e) GFS between $\pm 30^{\circ}$ latitude; (f) GFS $\pm 45^{\circ}$ latitude. For the corresponding parameters, refer to Table $2 \mathrm{c}$.

which for universal multifractals, with $q<q_{c}$ yields:

$M_{q}^{\prime}=\lambda^{C_{1}}$

i.e., $\log M_{q}^{\prime}=C_{1} \log \lambda$ so that all the curves for the different moments "collapse" onto a single $q$-independent curve. Such plots are interesting because, on a single plot, we can independently evaluate both the scaling (the straightness of the 

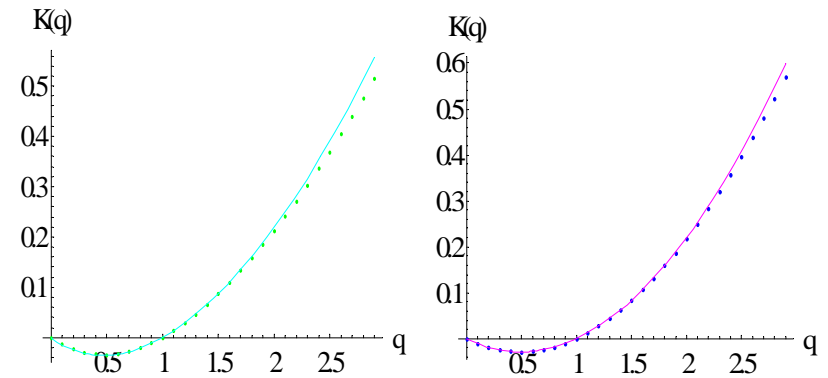

a)

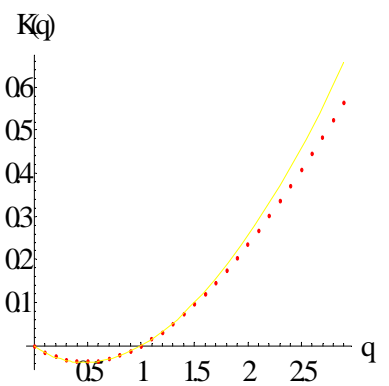

c)

Fig. 4. $K(q)$ for $T$ at $1000 \mathrm{mb} 00: 00 \mathrm{~h}$ timestep between $\pm 30^{\circ}$ for (a) GEM, (b) ERA40, (c) GFS. Dots are the calculated values for $K(q)$ at each $q\left(0.0\right.$ to 2.9). Solid lines are fits for (a) $C_{1}=0.125$, $\alpha=1.64$ (b), $C_{1}=0.113, \alpha=1.94$ and (c), $C_{1}=0.142, \alpha=1.72$. Notice the linear deviation of dots from the curves for $q>2$, which is indicative of a multifractal phase transition caused by the finite sample size.

collapsed lines) as well as the log-Levy nature of the generator - by the thinness of the collection of lines i.e. how well at a given scale the different moments collapse, how well they follow the functional form $\left(q^{\alpha}-q\right)$. If the flux follows Eq. (1b), it implies that the generator of the cascade (log flux) is a Levy variable, index $\alpha$, so that we may call this a "Levy collapse". The "thinness" can be quantified at each scale $\lambda$ by the relative standard deviation of $M_{q}$ ' as functions of $q$ :

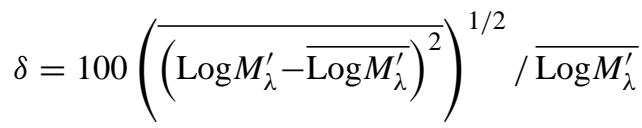

(the overbars indicate averaging with respect to the $q$ values). In Figs. 5, 6, 7 we see that the collapse for the different models, different fields, different altitudes, different forecast periods is very good, and this even out to scales where the scaling is not so well respected. The right hand column shows for each $\lambda$ the relative root mean square deviation $(=\delta)$ of the collapsed curves. For all scales the variations in $\log M_{q}^{\prime}$ are of the order $\pm 2- \pm 10 \%$ about the mean values (Fig. 5, 6, 7). The main exceptions are at the single small scale (largest $\lambda$ ) point which is presumably a finite size effect associated with the spatial discretization of the grid at the single pixel scale, as well as the occasionally deviations $>10 \%$ at the largest scales (where the number of independent structures sampled is lowest and the statistics are poorest). In Fig. 6, we show the systematic variation of the collapse with altitude, Fig. 7 with forecast time. The Levy collapse presentation has the attractive feature of allowing us to superpose the corresponding fields of the different models, thus making succinct inter model comparisons possible.

In the previous section, we noted that for all the fields, the regression estimates of $\alpha$ were close to the value 1.8. Looking more closely, we find overall that for a given field, all altitudes have roughly the same $\alpha$. There are some features worth mentioning; for example, for GEM, $\alpha$ is consistently about 0.20 smaller than ERA40 for $h_{s}$, while $u$ and $T$ at $1000 \mathrm{mb}$ have $\alpha \sim 1.65$ for GEM compared to 1.94 for ERA40 therefore show differences mostly not too different $(0.10$ greater $)$ for the higher altitudes. The GFS model typically shows intermediate results between the two other datasets.

In order to see how $\alpha$ varies for the same field but at different altitudes we calculated the "reduced moments," $M_{q}^{1 / C_{1}}$, with $C_{1}$ estimated numerically from $C_{1}=K^{\prime}(1)$. This separates the changes due to the mean intermittency $C_{1}$ from changes in the shape due to different $L_{\text {eff' }}$ 's and $\alpha$ 's. If the reduced moments are equal, then the only difference is in the mean intermittency $\left(C_{1}\right)$ parameter. Figure 6 shows the reduced moments for $u, T, h_{s}$ for GEM and ERA40 (the $48 \mathrm{~h}$ GEM forecast was very similar to the analysis) for $q=0.5$ and $q=2$. We see that the curves - including the small deviations from linearity at large scales (small $\lambda$ ) are very close so that variations in $C_{1}$ do indeed capture much of the field to field variability, variations in the value of $\alpha$ and $L_{\text {eff }}$ are indeed small.

\section{Conclusions}

Ever since Richardson speculated that atmospheric dynamics might be cascade-like, cascades have been regularly invoked. However, most meteorological applications do little more than identify a candidate cascade quantity, and then on the basis of dimensional arguments, determine (nonintermittent) spectral exponents; cascades are primarily reduced to conceptual aids rather than used as concrete models. Statistical mechanical-type arguments are sometimes further invoked to determine the direction of the cascade. In this way, turbulence theory plays a key role by identifying a priori the cascade quantity. The problem is that these theories have almost always been isotropic or (quasi isotropic) in either three or two spatial dimensions and have hence unnecessarily restricted the investigations of the cascade hypothesis. In the last 10 years, when this classical approach has been used to test cascades on large scale numerical weather models and related products (especially, Atmospheric GCM for the Earth Simulator, Hamilton et al., 2008 and ERA40, Straus and Ditlevsen, 1999), it has been found to fare rather poorly. The 

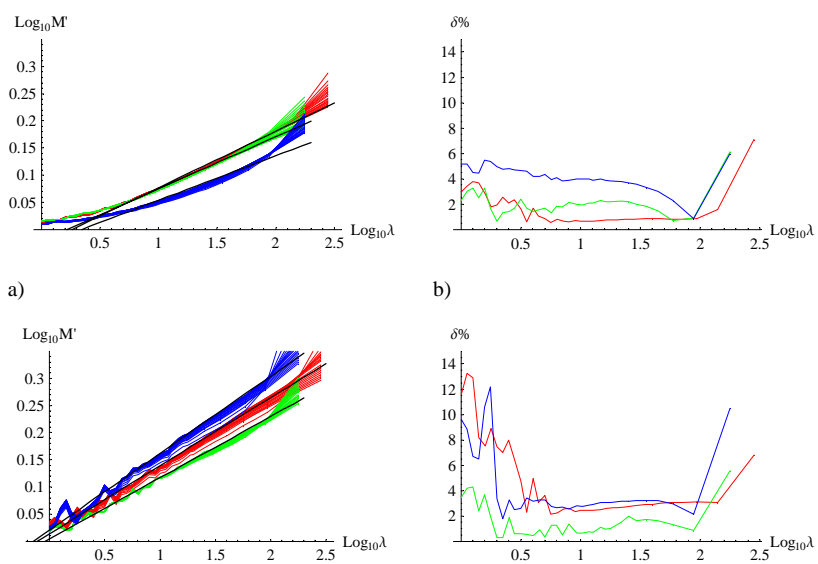

c)

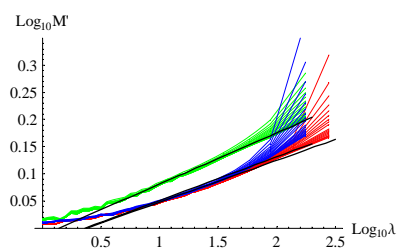

e)
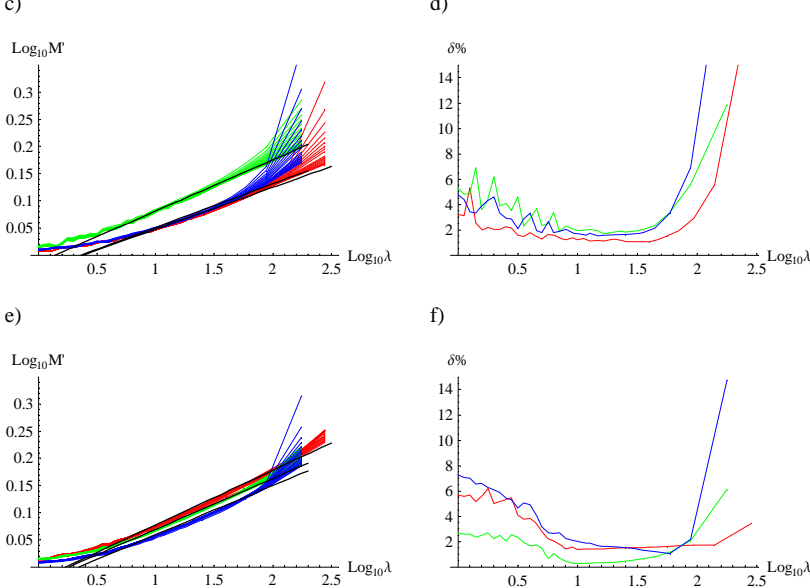

f)

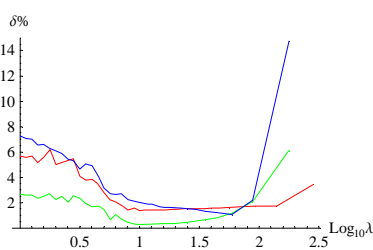

h)

Fig. 5. Levy Collapse Diagrams, showing $\log _{10} M_{q}$ '= $(\alpha-1) \log _{10} M_{q} /\left(q^{\alpha}-q\right), \quad$ showing moments, $\quad q=0.1$, $0.2, \quad 0.3, \quad \ldots 2.0, \quad$ (excluding $q=1.0$ ) for each diagram (left hand side), and their corresponding deviations $\delta=100\left(\overline{\left(\log M_{\lambda}^{\prime}-\overline{\log M_{\lambda}^{\prime}}\right)^{2}}\right)^{1 / 2} \overline{\log M_{\lambda}^{\prime}}$ as a function of scale (right hand size). If at a given scale $\lambda$, the curves overlap for all $q$ it implies that the generator $(\log )$ of the process is a Levy random variable with corresponding index $\alpha$; the "collapsed" log moments $=C_{1} \log \lambda$ (independent of $q$ ). In what follows, the red curves are GEM, green are ERA40, blue are GFS. (a) $u 700$ mbar: GEM (red, $\alpha=1.9$ ), ERA40 (green, $\alpha=2.0$ ), GFS (blue, $\alpha=1.85$ ); (b) percentage deviation of moments corresponding to (a); (c) $T$ 1000 mbar: GEM (red, =1.7), ERA40 (green, $\alpha=1.95$ ), GFS (blue, $\alpha=1.75$ ); (d) percentage deviation of moments corresponding to (c); (e) $T 700$ mbar: GEM (red, $\alpha=2.05$ ), ERA40 (green, $\alpha=2.2$ ), GFS (blue, $\alpha=2.15$ ); (f) percentage deviation of moments corresponding to (e); (g) $700 \mathrm{mbar} h_{s}$ : GEM (red, $\alpha=1.65$ ), ERA40 (green, $\alpha=1.8$ ), $h_{r}$ GFS (blue, $\alpha=1.8$ ); (h) percentage deviation of moments corresponding to $(\mathrm{g})$. The slope of the lines is $C_{1}$ and the horizontal intercept is the outer scale given in Table 2 .
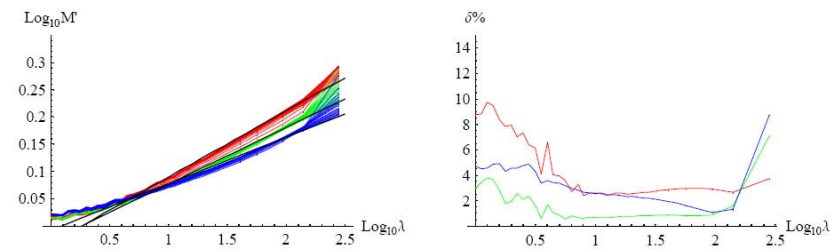

a)

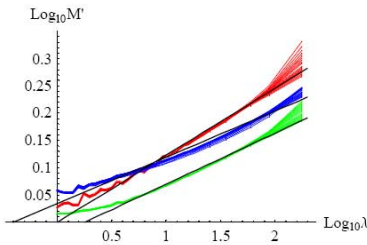

b)

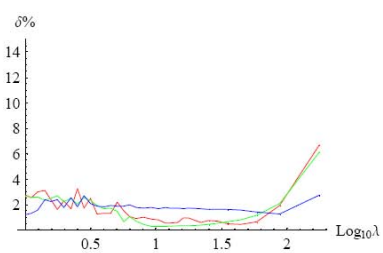

c)

d)

Fig. 6. More Levy collapse curves (left), here as a function of altitude, and their corresponding deviations $\delta$ (right) as a function of scale (a) GEM collapse curves $u$ : $1000 \mathrm{mb}$ (red, $\alpha=1.7$ ) , $700 \mathrm{mb}$ (green, $\alpha=1.9$ ), $200 \mathrm{mb}$ (blue, $\alpha=1.85$ ); (b) percentage deviation as a function of scale, corresponding to (a); (c) $h_{s}$ ERA40: collapse curves $1000 \mathrm{mb}$ (red, $\alpha=2.05$ ), $700 \mathrm{mb}$ (green, $\alpha=1.8$ ), $200 \mathrm{mb}$ (blue, $\alpha=1.73$ ); (d) percentage deviation as a function of scale, corresponding to (c). The slope of the lines is $C_{1}$ and the horizontal intercept is the outer scale given in Table 2 .
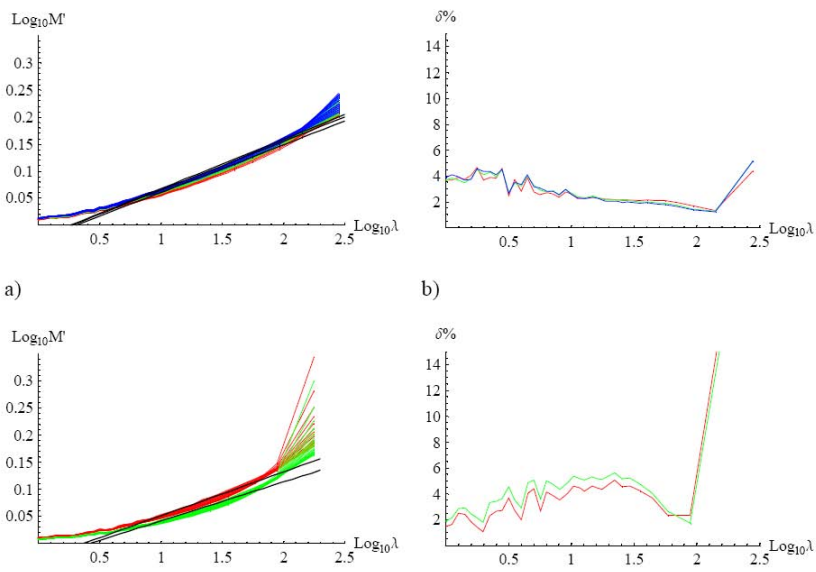

c)

d)

Fig. 7. More Levy collapse curves, (left) here as a function of forecast time, and their corresponding deviations $\delta$ as a function of scale (right) (a) GEM $h_{s} 850 \mathrm{mb}: t=0 \mathrm{~h}$ (red, $\alpha=1.73, C_{1}=0.90, \lambda=0.31$ ), $t=48 \mathrm{~h}$ (green, $\alpha=1.77, C_{1}=0.088, \lambda=0.27$ ), $t=144 \mathrm{~h}$ (blue, $\alpha=1.78$, $C_{1}=0.094, \lambda=0.26$ ); (b) percentage deviation as a function of scale, corresponding to a; (c) $h_{s}$ ERA40 collapse curves: $1000 \mathrm{mb}$ (red, $\alpha=2.00$ ), $700 \mathrm{mb}$ (green, $\alpha=1.97$ ); (d) percentage deviation as a function of scale, corresponding to (c). The slope of the lines is $C_{1}$ and the horizontal intercept is the outer scale, which is given in Table 2 for (b). Note that the $\alpha$ value may vary slightly depending on the fitting method. 
consequence is that there is no currently accepted turbulence interpretation of the model statistics.

However starting in the 1960's - in order to help understand intermittency, - precise, explicit multiplicative cascades models were developed. These phenomenological models are designed to reproduce some of the symmetries of the governing dynamical equations, specifically the scale by scale conservation of turbulent fluxes, the scale invariance symmetries of the dynamics and localness in Fourier space (so that interactions are primarily between structures of similar size). An advantage of directly exploiting the scale invariance is that no specific a priori assumptions need to be made about either isotropy or about the physical nature of the cascade quantity; the (generally anisotropic) scale symmetries play a crucial role. It is now understood that the implications are quite generic - they are insensitive to many details so that the statistics are expected to obey the basic prediction of multiplicative cascades Eq. (1a). In addition, due to the existence of stable attractive universality classes (a kind of multiplicative central limit theorem), there is an even more precise prediction - that the exponent $K(q)$ should depend only on two basic parameters (Eq. 1b).

Using (now standard) data analysis techniques, we demonstrate that three leading numerical models of the atmosphere accurately follow the predictions of multiplicative cascade models, including one (the reanalysis ERA 40) where extensive analysis of the classical (isotropic turbulence) approach failed to follow the predictions of the isotropic theories (Straus and Ditlevsen, 1999). In retrospect, it is fortunate that the models nearly perfectly follow the cascade predictions because an increasing number of analyses of empirical atmospheric data find that the atmosphere also has a cascade structure, so that the statistics of the data and numerical models are at least in qualitative (structural) agreement. The cascade structure of the intialisation fields makes it possible that the results are at least partially imposed by the constraint that they are near the (cascade-like) data at $t=0$. However, the forecasts - both short ( $48 \mathrm{~h})$ and medium-range (144h) - show that the model statistics are little changed although there is a small increase in the residuals, $\delta$. This suggests - but does not prove - that if the same models were run in "climate-mode" - i.e. for very long integrations - that they would maintain a cascade structure.

Over the period where numerical and multiplicative cascade models were developed in parallel, they have sometimes seemed irreconcilable - if only because the former are deterministic with strong scale truncations, whereas the latter are stochastic over arbitrarily wide ranges of scale. However in the last 15 years, with the development of "ensemble" forecasting (e.g., Zoth and Kalnay, 1993), there has been a revolution in attitudes about forecasting; it is now increasingly accepted that the goal is no longer a deterministic forecast of the weather (or climate) but rather the production of a distribution of possible future atmospheric states including their relative probabilities: today the aim is a stochas-

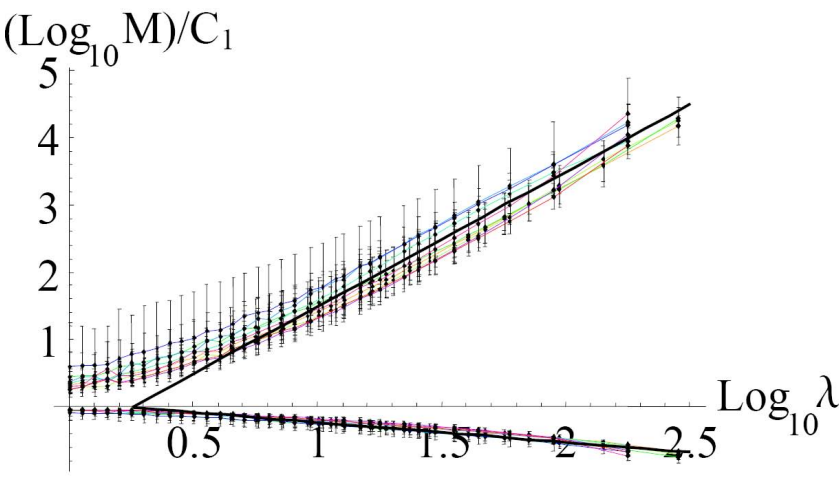

Fig. 8. "Reduced" $\mathrm{C}_{1}$-normalized moments $\left(M_{q}^{1 / C_{1}}\right)$ between $\pm 30^{\circ}$ latitude of order $q=0.5$ and 2 (bottom and top, respectively). Moments for ERA40 $u$ (teal), ERA40 $T$ (cyan), ERA40 $h_{s}$ (blue), GEM $u$ (orange), GEM $T$ (lime), GEM $h_{s}$ (green), GFS $u$ (purple), GFS $T$ (magenta), GFS $h_{r}$ (red), and reference lines for a cascade (black) with $L_{\mathrm{eff}}=11200 \mathrm{~km}$. The solid coloured lines are the mean value of the moments of a field over all pressure levels $(1000,850,700,500,200 \mathrm{mb})$, while the error bars show the spread (maximum/minimum) in values for different altitudes.

tic one. At the moment, this goal can only be achieved by a nontrivial marriage between the deterministic models and stochasticity, which is currently artificially introduced via various methods of generating initial conditions (e.g. "ensemble breeding", see Corazza et al., 2003). Other ad hoc attempts such as Buizza et al. (1999) and Palmer (2001) to introduce the required stochasticity include attempts at subgrid "stochastic parameterization". With the findings of this paper that cascades accurately describe the stochastic structure of the equations, several new avenues for modelling appear. The stochastic parameterization in this case can be used to properly implement an ensemble forecast - so differences in each member of the ensemble appear because of changes in the fields due to the stochastic parameterization. In the short term, using the cascades as theoretically "clean" subgrid parameterizations is promising, while in the medium to long term, quite new more direct purely stochastic forecasting techniques will be possible by exploiting the long range memory implicit in the cascade structures as mentioned in Scherzter and Lovejoy (2004).

Acknowledgements. We would like to acknowledge NSERC for Jonathan Stolle's scholarship support as well as Michel Bourqui, Andrew Ryzkhov, and Charles Lin for their helpful discussions and technical expertise. The analysis of GEM data was based on Environment Canada data and the ERA40 data developed by the ECMWF was obtained through UCAR. We also thank anonymous referees of Phys. Rev. Lett. and Physica A for some of their comments. This project did not receive any specific funding.

Edited by: A. Turiel

Reviewed by: A. M. Tarquis and two other anonymous referees 


\section{References}

Biferale, L.: Shell models of energy cascade turbulence, Annu. Rev. Fluid Mech., 35, 441-468, doi:10.1146/annurev.fluid.35.101101.161122, 2003.

Blender, R. and Fraedrich, K.: Long time memory in global warming simulations, Geophys. Res. Lett., 30, 1769-1772, doi:10.1029/2003GL017666, 2003.

Boer, G. J. and Shepherd, T. G.: Large-scale two-dimensional turbulence in the atmosphere, J. Atmos. Sci., 40, 164-184, 1983.

Buizza, R., Miller, M., and Palmer, T. N.: Stochastic representation of model uncertainties in the ECMWF Ensemble Prediction System, Q. J. Roy. Meteor. Soc., 125, 2887-2908, 1999.

Chigirinskaya, H. Y. and Schertzer, D.: Cascade of scaling gyroscopes: Lie stucture, universal multifractals and self-organized criticality in turbulence, in: Stochastic Models in Geosystems, edited by: Molchanov, S. A. and Woyczynski, W. A., Springer, New York, 57-81, 1997.

Chigirinskaya, Y., Schertzer, D., and. Lovejoy, S: An alternative to shell-models, more complete and yet simple models of intermittency, in: European Turbulence 7, edited by: Frisch, U., Kluwer Academic Press, Dordrecht, 263-266, 1998.

Cho, J. and Lindborg, E.: Horizontal velocity structure functions in the upper troposphere and lower stratosphere I: Observations, J. Geophys. Res., 106,, 10223-10232, 2001.

CMC Global Data Assimilation System (DAS), online available at: http://www.meted.ucar.edu/nwp/pcu2/GEMglobal/ gemglobassim.htm, last access: 22 July 2009.

Corazza, M., Kalnay, E., Patil, D. J., Yang, S.-C., Morss, R., Cai, M., Szunyogh, I., Hunt, B. R., and Yorke, J. A.: Use of the breeding technique to estimate the structure of the analysis "errors of the day", Nonlin. Processes Geophys., 10, 233-243, 2003, http://www.nonlin-processes-geophys.net/10/233/2003/.

Côte, J., Desmarais, J.-D., Gravel, S., Methot, A., Patoine, A., Roch, M., and Staniforth, A.: The operational CMC-MRB global environmental multiscale (GEM) model. Part II: Results, Mon. Weather Rev., 126, 1397-1418, 1998.

Côte, J., Gravel, S., Methot, A., Patoine, A., Roch, M., and Staniforth, A.: The operational CMC-MRB global environmental multiscale (GEM) model. Part I: Design considerations and formulation, Mon. Weather Rev., 126, 1373-1395, 1998.

Dewan, E. and Good, R.: Saturation and the universal spectrum for vertical profiles of horizontal scalar winds in the atmosphere, J. Geophys. Res., 91, 2742-2748, 1986.

Dewan, E.: Saturated-cascade similitude theory of gravity wave spectra, J. Geophys. Res., 102, 29799-29817, 1997.

Fraedrich, K. and Blender, R.: Scaling of Atmosphere and Ocean Temperature Correlations in Observations and Climate Models, Phys. Rev. Lett., 90, 108501, doi:10.1103/PhysRevLett.90.108501, 2003.

Frisch, U., Kurien, S., Pandit, R., Pauls, W., Ray, S. S., Wirth, A., and Zhu, J.-Z.: Hyperviscosity, Galerkin Truncation, and Bottlenecks in Turbulence, Phys. Rev. Lett., 101, 144501, doi:0.1103/PhysRevLett.101.144501, 2008.

Gage, K. S. and Nastrom, G. D.: Theoretical interpretation of atmospheric wavenumber spectra of wind and temperature observed by commercial aircraft during GASP, J. Atmos. Sci., 43, 729$740,1986$.

Gagnon, J.-S., Lovejoy, S., and Schertzer, D.: Multifractal earth topography, Nonlin. Processes Geophys., 13, 541-570, 2006, http://www.nonlin-processes-geophys.net/13/541/2006/.

Gardner, C.: Diffusive filtering theory of gravity wave spectra in the atmosphere, J. Geophys. Res., 99, 20601-20622, 1994.

Hamilton, K., Takahashi, Y. O., and Ohfuchi, W.: Mesoscale spectrum of atmospheric motions investigated in a very fine resolution global general ciruculation model, J. Geophys. Res., 113, D18110, doi:18110.11029/12008JD009785, 2008.

Hamilton, K.: Numerical Resolution and Modeling of the Global Atmospheric Circulation: A Review of Our Current Understanding and Outstanding Issues in High Resolution Numerical Modelling of the Atmosphere and Ocean, edited by: Hamilton, K. and Ohfuchi, W., 7-27, 2007.

Haugen, N. E. L. and Brandenburg, A.: Inertial range scaling in numerical turbulence with hyperviscosity, Phys. Rev. E, 026405, doi:10.1103/PhysRevE.70.026405, 2004.

High-resolution CMC GRIB dataset, online available at: http: //www.weatheroffice.gc.ca/grib/High-resolution_GRIB_e.html, last access: 6 February 2009.

Kaneda, Y., Ishihara, T., Yokokawa M., Itakura K., and Uno, A.: Energy dissipation rate and energy spectrum in high resolution direct numerical simulations of turbulence in a periodic box, Phys. Fluids, 15, L21-L24, doi:10.1063/1.1539855, 2003.

Kolmogorov, A. N.: Local structure of turbulence in an incompressible liquid for very large Reynolds numbers, Proc. Acad. Sci. USSR. Geochem Sect., 30, 299-303, 1941.

Landau, L. D. and Lifshitz, E. M.: Fluid Mechanics, 2nd Ed., Course of Theoretical Physics, Vol. 6, Pergamon, Oxford, 1987.

Lilley, M., Lovejoy, S., Strawbridge, K., and Schertzer D.: 23/9 dimensional anisotropic scaling of passive admixtures using lidar aerosol data, Phys. Rev. E, 70, 036307-1, doi:10.1103/PhysRevE.70.036307, 2004.

Lilley, M. Lovejoy, S., Strawbridge, K. B., Schertzer, D., and Radkevich, A.: Scaling turbulent atmospheric stratification, Part II: spatial stratification and intermittency from lidar data, Q. J. Roy. Meteor. Soc. 134, 301-315, doi:10.1002/qj.1202, 2008.

Lindborg, E.: Can the atmospheric kinetic energy spectrum be explained by two-dimensional turbulence?, J. Fluid Mech., 388, 259-288, 1999.

Lovejoy, S., Schertzer, D., and Stanway, J. D.: Direct Evidence of planetary scale atmospheric cascade dynamics, Phys. Rev. Lett. 86, 5200-5203, 2001.

Lovejoy, S. Schertzer, D., and Tuck, A. F.: Fractal aircraft trajectories and nonclassical turbulent exponents, Phys. Rev. E, 70, 036306-1, doi:0.1103/PhysRevE.70.03630, 2004.

Lovejoy, S., Hovde, S., Tuck, A., and Schertzer, D.: Is isotropic turbulence relevant in the atmosphere?, Geophys. Res. Lett., 34, L14802, doi:10.1029/2007GL029359, 2007.

Lovejoy, S., Schertzer, D., Lilley, M., Strawbridge, K., and Radkevich, A.: Scaling turbulent atmospheric stratification. I: turbulence and waves, Q. J. Roy. Meteor. Soc., 134, 277-300, doi:10.1002/qj.1201, 2008.

Lovejoy, S., Schertzer, D., Allaire, V., Bourgeois, T., King, S., Pinel, J., and Stolle, J.: Atmospheric complexity or scale by scale simplicity?, Geophys. Res. Lett., 36, L01801, doi:01810.01029/02008GL035863, 2009a.

Lovejoy, S. and Schertzer, D.: Towards a new synthesis for atmospheric dynamics: space-time cascades, Atmos. Res., in press, 2009.

Lovejoy, S., Tuck, A., and Schertzer, D.: The horizontal cascade 
structure of the atmosphere from aircraft measurements, J. Geophys. Res., submitted, 2009b.

Lovejoy, S., Tuck, A. F., Schertzer, D., and Hovde, S. J.: Reinterpreting aircraft measurements in anisotropic scaling turbulence, Atmos. Chem. Phys., 9, 5007-5025, 2009c, http://www.atmos-chem-phys.net/9/5007/2009/.

Lovejoy, S., Tuck, A. F., Hovde S. J., and Schertzer D.: The vertical cascade structure of the atmosphere and multifractal drop sonde outages, J. Geophys. Res., 114, D07111, doi:10.1029/2008JD010651, 2009d.

Mandelbrot, B. B.: Intermittent turbulence in self-similar cascades: divergence of high moments and dimension of the carrier, J. Fluid Mech., 62, 331-358, 1974.

Monin, A. S. and Yaglom, A. M.: Statistical Fluid Mechanics, MIT Press, Boston, 1975.

NCEP Office Note 442: The GFS atmospheric model, online available at: http://www.emc.ncep.noaa.gov/officenotes/newernotes/ on442.pdf, last access: 6 February 2009.

Novikov, E. A. and Stewart, R.: Intermittency of turbulence and spectrum of fluctuations in energy dissipation, Izvestiia Akademii nauk SSSR, Seriia Geofizicheskaia, 3, 408-412, 1964.

Okamoto, K. and Derber, J.: Assimilation of SSM/I radiance in the NCEP global data assimilation system, Mon. Weather Rev., doi: 10.1175/MWR3205.1, 2006.

Orszag, S. A. and Patterson, G. S.: Numerical simulation of threedimensional homogenous Turbulence, Phys. Rev. Lett., 28, 7679, 1972.

Parisi, G. and Frisch, U.: A multifractal model of intermittency, in: Turbulence and predictability in geophysical fluid dynamics and climate dynamics, edited by: Ghil, M., Benzi, R., and Parisi, G., Amsterdam, Netherlands, 84-88, 1985.

Palmer, T. N.: A nonlinear dynamical perspective on model error: A proposal for non-local stochastic-dynamic parametrization in weather and climate prediction models, Q. J. Roy. Meteor. Soc., 127, 279-304, 2001.

Richardson, L. F.: Weather Prediction by Numerical Process, Cambridge University Press, Cambridge, United Kingdom, 1922, republished by Dover, New York, 1965.

Richardson, L. F.: Atmospheric diffusion shown on a distanceneighbour graph, Proc. Roy. Soc. Lond. A Mat., 110, 709-737, 1926.

Royer, J. F., Biaou, A., Chauvin, F., Schertzer, D., and Lovejoy, S.: Multifractal analysis of the evolution of simulated precipitation over France in a climate scenario, Comptes Rendues Geophysiques, 340, 431-440, 2008.

Schertzer, D. and Lovejoy, S.: The dimension and intermittency of atmospheric dynamics, in: Turbulent Shear Flow 4 7, edited by: Launder, B., Springer, Berlin, 1985.

Schertzer, D. and Lovejoy, S.: Physical modeling and analysis of rain and clouds by anisotropic scaling of multiplicative processes, J. Geophys. Res., 92, 9692-9714, 1987.

Schertzer, D. and Lovejoy, S.: Uncertainty and Predictability in Geophysics: Chaos and Multifractal Insights, in: State of the Planet, Frontiers and Challenges in Geophysics, edited by: Sparks, R. S. J. and Hawkesworth, C. J., AGU, Washington, 317334, 2004
Schertzer, D., Lovejoy, S., and Lavallée, D.: Generic multifractal phase transitions and self-organized criticality in Cellular automata: prospects, in: Astronomy and astrophysics, edited by: Perdang J. M. and Lejeune, A., World Scientific, 216-227, 1993.

Sela, J. G.: The NMC spectral model, NOAA technical report NWS-30, 1982.

Sela, J. G.: The new NMC operational spectral model, in: Eighth Conference on Numerical Weather Prediction, Baltimore, Maryland, 22-26 February 1988.

Steinberg, H. L., Wiin-Nielsen, A., and Yang C.-H.: On nonlinear cascades in atmospheric flows, J. Geophys. Res. 76, 8629-8640, 1971.

Stolle, J.: A spatial and temporal stochastic cascade analysis of meteorological models and reanalyses, McGill University, Canada, 2009.

Stolle, J., Lovejoy, S., and Scherzter, D.: The temporal cascade structure and space-time relations for reanalyses and Global Circulation models, in preparation, 2009.

Straus, D. M. and Ditlevsen, P.: Two dimensional turbulence properties of the ECMWF, Tellus, 51A, 749-772, 1999.

Syroka, J. and Toumi, R.: Scaling and persistence in observed and modelled surface temperature, Geophys. Res. Lett., 28, 32553255, 2001.

Toth, Z. and Kalnay, E.: Ensemble forecasting at NMC: the generation of perturbations, B. Am. Meteorol. Soc., 74, 2317-2330, 1993.

Uppala, S. M., Kallberg, P. W., Simmons, A. J., Andrae, U., Da Costa Bechtold, V., Fiorino, M., Gibson, J. K., Haseler, J., Hernandez, A., Kelly, G. A., Li, X., Onogi, K., Sarinen, S., Sokka N., Allan, R. P., Anderson, E., Arpe, K., Balmaseda, M. A., Beljaars, A. C. M., Van de Berg, L., Bidloti, J., Bormann, N., Caires, S., Chevallier, F., Dethof, A., Dragosavac, M., Fisher, M., Fuentes, M., Hagemann, S., Holm, E., Hoskins, B. J., Isaksen, L., Janssen, P. A. E. M., Jenne, R., McNally, A. P., Mahfouf, J.-F., Morcrette, J.-J., Rayner, N. A., Saunders, R. W., SIMON, P., Sterl, A., Trenberth, K. E., Untch, A., Vasiljevic, D., Viterbo, P., and Woollen, J.: The ERA40 re-analysis, Q. J. Roy. Meteor. Soc., 131, 2961-3012, doi:10.1256/qj.04.176, 2005.

Van Zandt, T. E.: A universal spectrum of buoyancy waves in the atmosphere, Geophys. Res. Lett., 9, 575-578, 1982.

Vincent, A. and Meneguzzi, M.: The spatial structure and statistical properties of homogeneous turbulence, J. Fluid Mech., 225, 120, 1991.

Yaglom, A. M.: The influence of fluctuations in energy dissipation on the shape of turbulence characteristics in the inertial interval, Soviet Physics - Doklady., 2, 26-29, 1966.

Yokokawa, M., Itakura, K., Uno, A., Ishihara, T., and Kaneda, Y.: 16.4-Tflops Direct Numerical Simulation of Turbulence by a Fourier Spectral Method on the Earth Simulator, in: SC2002, Baltimore, USA, 16-22 November, online available at: http://www.sc-2002.org/paperpdfs/pap.pap273.pdf, 2002. 